\title{
Absolute Magnitude Calibration for Dwarfs Based on the Colour-Magnitude Diagrams of Galactic Clusters
}

\author{
S. Karaali ${ }^{1,2}$, E. Yaz Gökçe ${ }^{1}$, S. Bilir ${ }^{1}$ and S. Tunçel Güçtekin ${ }^{1}$ \\ ${ }^{1}$ Department of Astronomy and Space Sciences, Faculty of Science, Istanbul University, 34119, Istanbul, Turkey \\ 2Email: karsa@istanbul.edu.tr
}

(ReCeIved December 30, 2013; ACCEPTEd May 22, 2014)

\begin{abstract}
We present two absolute magnitude calibrations for dwarfs based on colour-magnitude diagrams of Galactic clusters. The combination of the $M_{g}$ absolute magnitudes of the dwarf fiducial sequences of the clusters M92, M13, M5, NGC 2420, M67, and NGC 6791 with the corresponding metallicities provides absolute magnitude calibration for a given $(g-r)_{0}$ colour. The calibration is defined in the colour interval $0.25 \leq(g-r)_{0} \leq 1.25 \mathrm{mag}$ and it covers the metallicity interval $-2.15 \leq[\mathrm{Fe} / \mathrm{H}] \leq+0.37 \mathrm{dex}$. The absolute magnitude residuals obtained by the application of the procedure to another set of Galactic clusters lie in the interval $-0.15 \leq \Delta M_{g} \leq+0.12$ mag. The mean and standard deviation of the residuals are $\left\langle\Delta M_{g}\right\rangle=-0.002$ and $\sigma=0.065 \mathrm{mag}$, respectively. The calibration of the $M_{J}$ absolute magnitude in terms of metallicity is carried out by using the fiducial sequences of the clusters M92, M13, 47 Tuc, NGC 2158, and NGC 6791. It is defined in the colour interval $0.90 \leq(V-J)_{0} \leq 1.75 \mathrm{mag}$ and it covers the same metallicity interval of the $M_{g}$ calibration. The absolute magnitude residuals obtained by the application of the procedure to the cluster M5 $([\mathrm{Fe} / \mathrm{H}]=-1.40 \mathrm{dex})$ and 46 solar metallicity, $-0.45 \leq[\mathrm{Fe} / \mathrm{H}] \leq+0.35 \mathrm{dex}$, field stars lie in the interval -0.29 and +0.35 mag. However, the range of $87 \%$ of them is rather shorter, $-0.20 \leq \Delta M_{J} \leq+0.20 \mathrm{mag}$. The mean and standard deviation of all residuals are $\left\langle\Delta M_{J}\right\rangle=0.05$ and $\sigma=0.13 \mathrm{mag}$, respectively. The derived relations are applicable to stars older than $4 \mathrm{Gyr}$ for the $M_{g}$ calibration, and older than $2 \mathrm{Gyr}$ for the $M_{J}$ calibration. The cited limits are the ages of the youngest calibration clusters in the two systems.

Keywords: globular clusters: individual (M5, M13, M92, 47 Tuc) - (Galaxy:) open clusters: individual (M67, NGC 2158, NGC 2420, NGC 6791) - (stars:) dwarfs - stars: general - stars: late-type - techniques: photometric (Galaxy:)
\end{abstract}

\section{INTRODUCTION}

The distance of an astronomical object plays an important role in deriving absolute magnitudes of stars and determining the three-dimensional structure of our Galaxy. For nearby stars, Hipparcos (ESA 1997) is the main supplier of the data where a trigonometric parallax has been used. However, for stars at large distances, a photometric parallax is inevitable.

Different methods appeared in the literature related to absolute magnitude determination. Nissen \& Schuster (1991) and Laird, Carney, \& Latham (1988) used the offset from standard main-sequence for the absolute magnitude determination of dwarfs. The studies of Phleps et al. (2000) and Chen et al. (2001) are based on the colour-absolute magnitude diagrams of some specific clusters whose metal abundances are adopted as representative of a Galactic population, i.e., thin and thick discs, and halo. Siegel et al. (2002) derived two relations, one for solar-like abundances and another for

$\dagger$ Retired. metal-poor stars, between the $M_{R}$ absolute magnitude and $R-I$ colour index which provide absolute magnitude estimation of dwarfs with different metallicities by means of linear interpolation or extrapolation.

A more recent procedure for absolute magnitude estimation for dwarfs and giants simultaneously is based on the measured atmospheric parameters and the time spent by the star in each region of the $\mathrm{H}-\mathrm{R}$ diagram. For details of this procedure, we refer to the works of Breddels et al. (2010) and Zwitter et al. (2010).

The most recent absolute magnitude calibrations are those of Karaali, Bilir, \& Yaz Gökçe (2012) and Karaali, Bilir \& Yaz Gökçe (2013a, 2013b). Karaali et al. combined the colour-apparent magnitude diagrams of Galactic clusters with different metal abundances and their distance moduli, and calibrated the $M_{V}, M_{g}, M_{J}$, and $M_{K_{s}}$ absolute magnitudes of red giants in terms of metallicity. Here, we will apply the same procedure to the dwarfs using the Sloan Digital Sky Survey (SDSS; York et al. 2000) and Two Micron All 
Table 1. Data for the clusters used in the calibration with SDSS.

\begin{tabular}{lcccr}
\hline \hline Cluster & $\begin{array}{c}E(B-V) \\
(\mathrm{mag})\end{array}$ & $\begin{array}{c}\mu_{0} \\
(\mathrm{mag})\end{array}$ & $\begin{array}{c}{[\mathrm{Fe} / \mathrm{H}]} \\
(\mathrm{dex})\end{array}$ & Ref \\
\hline M92 & 0.025 & 14.72 & -2.15 & 1 \\
M13 & 0.020 & 14.38 & -1.41 & 1 \\
M5 & 0.040 & 14.37 & -1.26 & 2 \\
NGC 2420 & 0.040 & 12.00 & -0.37 & 2 \\
M67 & 0.038 & 9.53 & -0.04 & 1 \\
NGC 6791 & 0.150 & 13.10 & +0.37 & 3 \\
\hline \hline
\end{tabular}

(1) Gratton et al. (1997), (2) An et al. (2008), (3) Brasseur et al. (2010)

Sky Survey (2MASS; Skrutskie et al. 2006) data, two of the widely used photometric systems in Galactic research. This procedure is different than the ones in Karaali et al. (2003) and Karaali, Bilir, \& Tunçel (2005) where the absolute magnitude was calibrated in terms of the parameter $\delta_{0.6}$, the ultraviolet excess relative to the Hyades cluster, reduced to the intrinsic colour $B-V=0.60 \mathrm{mag}$. The $U$ ultraviolet magnitude cannot be observed accurately for each star. Whereas, various procedures can be utilized to determine metallicity for a larger set of stars. Hence, for the procedure in this paper, we expect an advantage over the former one.

This paper is the fourth one related to the calibration of absolute magnitudes of stars. The former ones were devoted to red giants, while in this paper the subject is dwarfs. We calibrate the $M_{g}$ and $M_{J}$ absolute magnitudes in terms of metallicity. The outline of the paper follows the presentation of the data in Section 2, procedure utilized for calibration in Section 3, and a summary and discussion in Section 4.

\section{DATA}

We calibrated two different absolute magnitudes, $M_{g}$ and $M_{J}$, in terms of metallicity. Hence, we used two different sets of data. The calibration of $g_{0}$ with $(g-r)_{0}$ is given in Section 2.1 , while that for $J_{0}$ with $(V-J)_{0}$ is presented in Section 2.2 .

\subsection{Data for calibration with SDSS: $g_{0} \times(g-r)_{0}$}

Our sample consists of M92, M13, M5, NGC 2420, M67, and NGC 6791 stellar clusters with different metal abundance. The SDSS photometric data of the clusters were taken from An et al. (2008), while the $\left(g-M_{g}\right)_{0}$ true distance modulus, $E(B-V)$ colour excess, and $[\mathrm{Fe} / \mathrm{H}]$ iron abundance are from the references in the last column of Table 1 . The range of the metallicity is $-2.15 \leq[\mathrm{Fe} / \mathrm{H}] \leq+0.37$ dex. The $g$ and $g-r$ fiducials of the stellar clusters adapted from An et al. (2008) are given in Table 2. As in our previous works, we used $R_{V}=3.1$ (Cardelli, Clayton, \& Mathis 1989) to obtain the total extinction at the $V$ band. Then, we used the equations of Fan (1999) to calculate the total extinction in $A_{g}$ and the selective absorption in SDSS, $E(g-r) / A_{V}=0.341$.
We fitted the $g_{0} \times(g-r)_{0}$ main-sequence of each cluster to a fifth-degree polynomial. The calibration of $g_{0}$ is as follows:

$$
g_{0}=\sum_{i=0}^{5} a_{i}(g-r)_{0}^{i}
$$

The numerical values of $a_{i}$ coefficients are given in Table 3 and the corresponding diagrams are shown in Figure 1. The $(g-r)_{0}$ values in the second line of the table denote the range of $(g-r)_{0}$ available for each cluster.

\subsection{Data for calibration with 2MASS: $J_{0} \times(V-J)_{0}$}

Five clusters with different metallicities, i.e., M92, M13, NGC 6791, 47 Tuc, and NGC 2158, were selected for our programme. The $J$ and $V-J$ data for the first three clusters were taken from Brasseur et al. (2010), while those for the clusters of 47 Tuc and NGC 2158 were transformed from the $B V R I$ and gri data, respectively, as explained in the following. For 47 Tuc, we de-reddened the $V, B-V$, and $R-I$ magnitude and colours of Alcaino et al. (1990) and transformed them to the $(V-J)_{0}$ colours by the following equation of Bilir et al. (2008):

$$
(V-J)_{0}=1.557(B-V)_{0}+0.461(R-I)_{0}+0.049 .
$$

Then, we combined the $V_{0}$ magnitude and $(V-J)_{0}$ colour and obtained the $J_{0}$ magnitude. For the cluster NGC 2158 , we obtained the $(V-J)_{0}$ colours in two steps. First, we transformed the de-reddened $g_{0},(g-r)_{0}$, and $(r-i)_{0}$ data of Smolinski et al. (2011) to the $(g-J)_{0}$ colour by using the following equation of Bilir et al. (2008):

$$
(g-J)_{0}=1.536(g-r)_{0}+1.400(r-i)_{0}+0.488 .
$$

Then, the magnitude $g_{0}$ is combined with this equation to give the $J_{0}$ magnitude, and further, Equation (2) of Chonis \& Gaskell (2008) given in the following:

$$
V_{0}=g_{0}-0.587(g-r)_{0}-0.011,
$$

is used in the evaluation of $(V-J)_{0}$ as follows:

$$
(V-J)_{0}=(g-J)_{0}-0.587(g-r)_{0}-0.011 .
$$

The range of the metallicity of the clusters given in iron abundance is $-2.15 \leq[\mathrm{Fe} / \mathrm{H}] \leq 0.37 \mathrm{dex}$. The true distance modulus, $\mu_{0}, E(B-V)$ colour excess, and $[\mathrm{Fe} / \mathrm{H}]$ iron abundance for the clusters are taken from the authors given in the last column of Table 4. Two references are given for the clusters 47 Tuc and NGC 2158. The metallicity of 47 Tuc is taken from Alcaino et al. (1990), while the colour excess and true distance modulus are from Harris 
Table 2. $g, r$ magnitudes, $g-r$, and de-reddened $(g-r)_{0}$ colours and $g_{0}$ magnitudes for the clusters M92, M13, M5, NGC 2420, M67, and NGC 6791 used in the calibration with SDSS.

\begin{tabular}{|c|c|c|c|c|c|c|c|c|c|}
\hline$r$ & $g-r$ & $g$ & $(g-r)_{0}$ & $g_{0}$ & $r$ & $g-r$ & $g$ & $(g-r)_{0}$ & $g_{0}$ \\
\hline \multicolumn{5}{|c|}{ M92 } & \multicolumn{5}{|c|}{ M5 } \\
\hline 18.750 & 0.225 & 18.975 & 0.199 & 18.882 & 19.850 & 0.411 & 20.261 & 0.369 & 20.112 \\
\hline 19.050 & 0.240 & 19.290 & 0.214 & 19.197 & 20.150 & 0.463 & 20.613 & 0.421 & 20.464 \\
\hline 19.350 & 0.263 & 19.613 & 0.237 & 19.520 & 20.450 & 0.522 & 20.972 & 0.480 & 20.823 \\
\hline 19.650 & 0.291 & 19.941 & 0.265 & 19.848 & 20.750 & 0.588 & 21.338 & 0.546 & 21.189 \\
\hline 19.950 & 0.326 & 20.276 & 0.300 & 20.183 & 21.050 & 0.665 & 21.715 & 0.623 & 21.566 \\
\hline 20.250 & 0.369 & 20.619 & 0.343 & 20.526 & 21.350 & 0.754 & 22.104 & 0.712 & 21.955 \\
\hline 20.550 & 0.422 & 20.972 & 0.396 & 20.879 & 21.650 & 0.850 & 22.500 & 0.808 & 22.351 \\
\hline 20.850 & 0.483 & 21.333 & 0.457 & 21.240 & \multicolumn{5}{|c|}{ NGC 2420} \\
\hline \multicolumn{5}{|c|}{ M13 } & 15.100 & 0.235 & 15.335 & 0.193 & 15.186 \\
\hline 18.750 & 0.270 & 19.020 & 0.249 & 18.946 & 15.300 & 0.246 & 15.546 & 0.204 & 15.397 \\
\hline 19.050 & 0.288 & 19.338 & 0.267 & 19.264 & 15.500 & 0.265 & 15.765 & 0.223 & 15.616 \\
\hline 19.350 & 0.315 & 19.665 & 0.294 & 19.591 & 15.700 & 0.290 & 15.990 & 0.248 & 15.841 \\
\hline 19.650 & 0.349 & 19.999 & 0.328 & 19.925 & 15.900 & 0.315 & 16.215 & 0.273 & 16.066 \\
\hline 19.950 & 0.391 & 20.341 & 0.370 & 20.267 & 16.100 & 0.340 & 16.440 & 0.298 & 16.291 \\
\hline 20.250 & 0.441 & 20.691 & 0.420 & 20.617 & 16.300 & 0.367 & 16.667 & 0.325 & 16.518 \\
\hline 20.550 & 0.500 & 21.050 & 0.479 & 20.976 & 16.500 & 0.398 & 16.898 & 0.356 & 16.749 \\
\hline 20.850 & 0.569 & 21.419 & 0.548 & 21.345 & 16.700 & 0.429 & 17.129 & 0.387 & 16.980 \\
\hline 21.150 & 0.643 & 21.793 & 0.622 & 21.719 & 16.900 & 0.463 & 17.363 & 0.421 & 17.214 \\
\hline 21.450 & 0.719 & 22.169 & 0.698 & 22.095 & 17.100 & 0.500 & 17.600 & 0.458 & 17.451 \\
\hline 21.750 & 0.798 & 22.548 & 0.777 & 22.474 & 17.300 & 0.539 & 17.839 & 0.497 & 17.690 \\
\hline \multicolumn{5}{|c|}{ M67 } & 17.500 & 0.586 & 18.086 & 0.544 & 17.937 \\
\hline 13.500 & 0.369 & 13.869 & 0.329 & 13.728 & 17.700 & 0.636 & 18.336 & 0.594 & 18.187 \\
\hline 13.700 & 0.388 & 14.088 & 0.348 & 13.947 & 17.900 & 0.689 & 18.589 & 0.647 & 18.440 \\
\hline 13.900 & 0.408 & 14.308 & 0.368 & 14.167 & 18.100 & 0.748 & 18.848 & 0.706 & 18.699 \\
\hline 14.100 & 0.431 & 14.531 & 0.391 & 14.390 & 18.300 & 0.812 & 19.112 & 0.77 & 18.963 \\
\hline 14.300 & 0.465 & 14.765 & 0.425 & 14.624 & 18.500 & 0.884 & 19.384 & 0.842 & 19.235 \\
\hline 14.500 & 0.507 & 15.007 & 0.467 & 14.866 & 18.700 & 0.958 & 19.658 & 0.916 & 19.509 \\
\hline 14.700 & 0.545 & 15.245 & 0.505 & 15.104 & 18.900 & 1.035 & 19.935 & 0.993 & 19.786 \\
\hline 14.900 & 0.579 & 15.479 & 0.539 & 15.338 & 19.100 & 1.120 & 20.220 & 1.078 & 20.071 \\
\hline 15.100 & 0.616 & 15.716 & 0.576 & 15.575 & 19.300 & 1.195 & 20.495 & 1.153 & 20.346 \\
\hline 15.300 & 0.661 & 15.961 & 0.621 & 15.820 & 19.500 & 1.255 & 20.755 & 1.213 & 20.606 \\
\hline 15.500 & 0.713 & 16.213 & 0.673 & 16.072 & 19.700 & 1.312 & 21.012 & 1.270 & 20.863 \\
\hline 15.700 & 0.772 & 16.472 & 0.732 & 16.331 & 19.900 & 1.360 & 21.260 & 1.318 & 21.111 \\
\hline 15.900 & 0.841 & 16.741 & 0.801 & 16.600 & 20.100 & 1.394 & 21.494 & 1.352 & 21.345 \\
\hline 16.100 & 0.912 & 17.012 & 0.872 & 16.871 & 20.300 & 1.422 & 21.722 & 1.380 & 21.573 \\
\hline 16.300 & 0.988 & 17.288 & 0.948 & 17.147 & 20.500 & 1.438 & 21.938 & 1.396 & 21.789 \\
\hline 16.500 & 1.070 & 17.570 & 1.030 & 17.429 & \multicolumn{5}{|c|}{ NGC 6791} \\
\hline 16.700 & 1.147 & 17.847 & 1.107 & 17.706 & 17.550 & 0.670 & 18.220 & 0.511 & 17.662 \\
\hline 16.900 & 1.216 & 18.116 & 1.176 & 17.975 & 17.750 & 0.682 & 18.432 & 0.523 & 17.874 \\
\hline 17.100 & 1.279 & 18.379 & 1.239 & 18.238 & 18.050 & 0.715 & 18.765 & 0.556 & 18.207 \\
\hline 17.300 & 1.330 & 18.630 & 1.290 & 18.489 & 18.350 & 0.760 & 19.110 & 0.601 & 18.552 \\
\hline 17.500 & 1.363 & 18.863 & 1.323 & 18.722 & 18.650 & 0.815 & 19.465 & 0.656 & 18.907 \\
\hline 17.700 & 1.392 & 19.092 & 1.352 & 18.951 & 18.950 & 0.886 & 19.836 & 0.727 & 19.278 \\
\hline 17.900 & 1.419 & 19.319 & 1.379 & 19.178 & 19.250 & 0.969 & 20.219 & 0.810 & 19.661 \\
\hline 18.100 & 1.440 & 19.540 & 1.400 & 19.399 & 19.550 & 1.061 & 20.611 & 0.902 & 20.053 \\
\hline \multicolumn{5}{|c|}{ M5 } & 19.850 & 1.162 & 21.012 & 1.003 & 20.454 \\
\hline 18.650 & 0.290 & 18.940 & 0.248 & 18.791 & 20.150 & 1.263 & 21.413 & 1.104 & 20.855 \\
\hline 18.950 & 0.308 & 19.258 & 0.266 & 19.109 & 20.450 & 1.354 & 21.804 & 1.195 & 21.246 \\
\hline 19.250 & 0.335 & 19.585 & 0.293 & 19.436 & 20.750 & 1.431 & 22.181 & 1.272 & 21.623 \\
\hline 19.550 & 0.368 & 19.918 & 0.326 & 19.769 & - & - & - & - & - \\
\hline
\end{tabular}


Table 3. The values of the coefficients $a_{i}(i=0, \ldots, 5)$ in Equation (1).

\begin{tabular}{lrrrrrr}
\hline \hline $\begin{array}{l}\text { Cluster } \\
(g-r)_{0} \text { interval }\end{array}$ & \multicolumn{1}{c}{ M92 } & M13 & \multicolumn{1}{c}{ M5 } & NGC 2420 & \multicolumn{1}{c}{ M67 } & NGC 6791 \\
& {$[0.20,0.46]$} & {$[0.25,0.78]$} & {$[0.25,0.81]$} & {$[0.19,1.40]$} & {$[0.33,1.40]$} & {$[0.51,1.27]$} \\
\hline$a_{5}$ & 7289.856 & 224.151 & 259.663 & 8.460 & 13.044 & 53.081 \\
$a_{4}$ & -12324.726 & -632.530 & -732.851 & -32.353 & -52.757 & -250.015 \\
$a_{3}$ & 8269.137 & 708.233 & 813.566 & 50.359 & 86.340 & 468.511 \\
$a_{2}$ & -2768.241 & -396.039 & -447.797 & -41.975 & -73.637 & -437.308 \\
$a_{1}$ & 472.894 & 116.688 & 128.576 & 23.339 & 37.436 & 207.802 \\
$a_{0}$ & -13.697 & 5.748 & 4.585 & 11.978 & 6.911 & -21.621 \\
\hline \hline
\end{tabular}

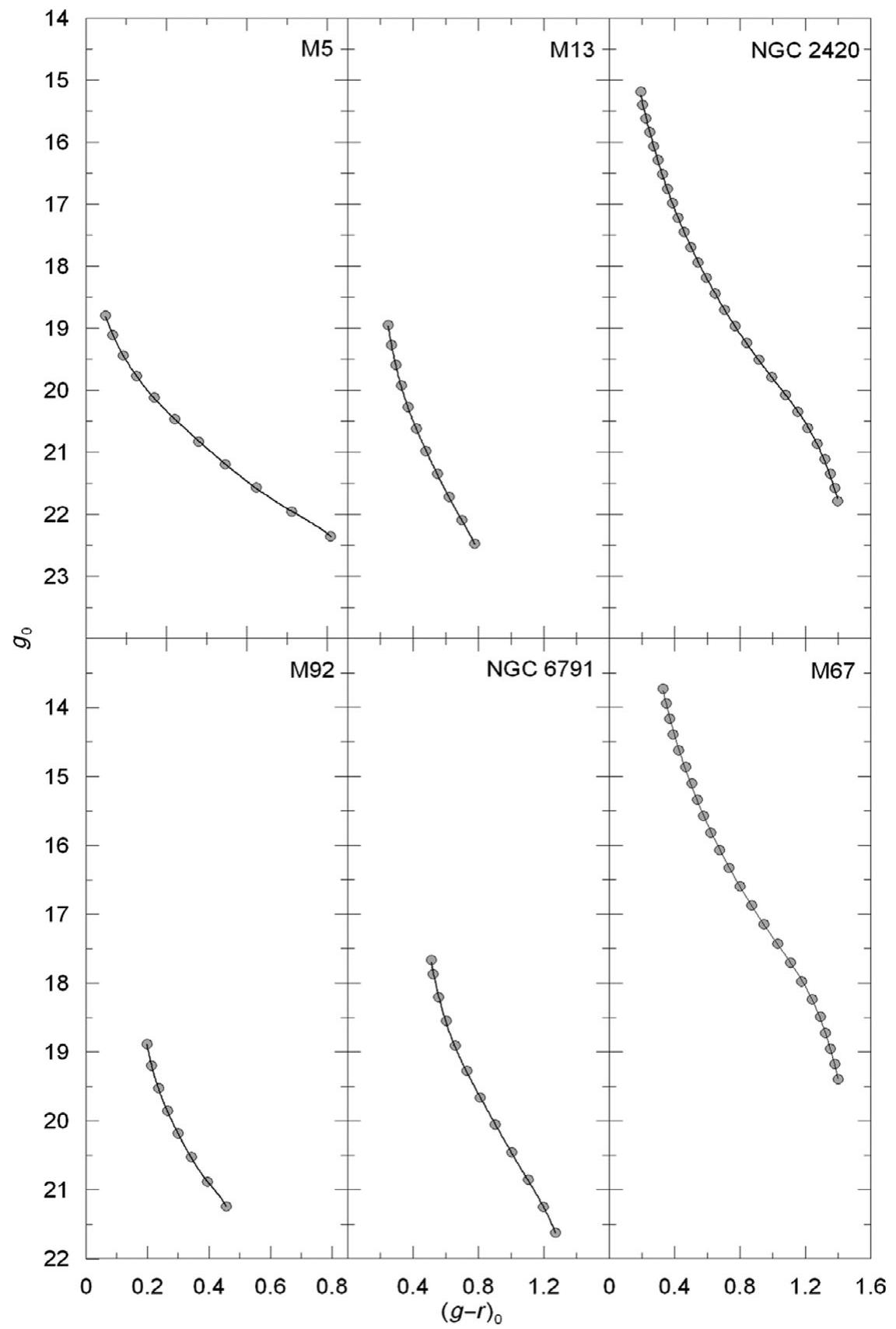

Figure 1. $g_{0} \times(g-r)_{0}$ colour-apparent magnitude diagrams of six stellar clusters used for the absolute magnitude calibration with SDSS. 


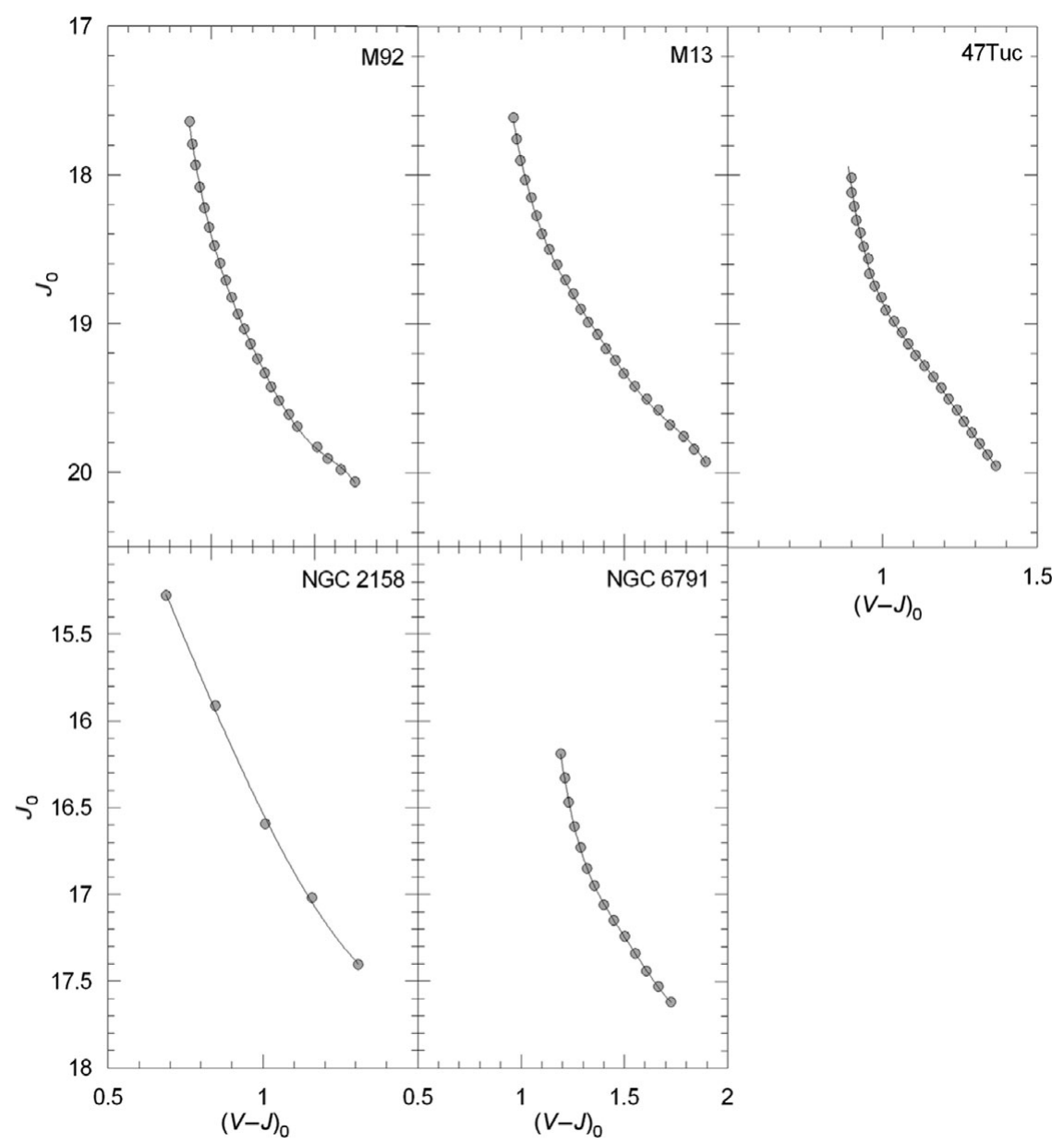

Figure 2. $J_{0} \times(V-J)_{0}$ colour-apparent magnitude diagrams for five clusters used for the $M_{J}$ absolute magnitude calibration.

Table 4. Data for the clusters used in the calibration with 2MASS.

\begin{tabular}{lcccc}
\hline \hline Cluster & $\begin{array}{c}E(B-V) \\
(\mathrm{mag})\end{array}$ & $\begin{array}{c}\mu_{0} \\
(\mathrm{mag})\end{array}$ & $\begin{array}{c}{[\mathrm{Fe} / \mathrm{H}]} \\
(\mathrm{dex})\end{array}$ & References \\
\hline M92 & 0.025 & 14.72 & -2.15 & 1 \\
M13 & 0.020 & 14.38 & -1.41 & 1 \\
47 Tuc & 0.220 & 15.40 & -1.27 & 2,3 \\
NGC 2158 & 0.440 & 13.38 & -0.25 & 4,5 \\
NGC 6791 & 0.150 & 13.10 & 0.37 & 6 \\
\hline \hline
\end{tabular}

(1) Gratton et al. (1997), (2) Harris (1996), (3) Alcaino et al. (1990), (4) Smolinski et al. (2011), (5) Arp \& Cuffey (1962), (6) Brasseur et al. (2010)

(1996). The metallicity and the colour excess of the cluster NGC 2158 are those of Smolinski et al. (2011), while its distance modulus is reduced from the apparent distance modulus in Arp \& Cuffey (1962), i.e., $\mu=14.74$ mag. Although this distance modulus is old, it provides $J_{0}$ and $(V-J)_{0}$ data which are more agreeable with the colours and magnitudes of the other clusters used in the calibration. The $J_{0}$ and $(V-J)_{0}$ data are presented in Table 5. We adopted the $R=A_{V} / E(B-V)=3.1$ to convert the colour excess to the extinction (Cardelli et al. 1989). Then, we used the equations $A_{J} / E(B-V)=0.87$ and $E(V-J) / E(B-V)=2.25$ (McCall 2004) to de-redden the $J$ magnitude and $V-J$ colour, respectively. The de-reddening of the $R-I$ colour used for the cluster 47 Tuc is carried out by the equation $E(R-I) / E(B-V)=0.60$ (Bilir et al. 2008).

We fitted the $J_{0} \times(V-J)_{0}$ sequence of four clusters to a fifth-degree polynomial, while a third-degree polynomial was sufficient for NGC 2158 due to less data. The calibration of $J_{0}$ is as follows:

$$
J_{0}=\sum_{i=0}^{5} b_{i}(V-J)_{0}^{i}
$$

The numerical values of the coefficients $b_{i} \quad(i=$ $0,1,2,3,4,5)$ are given in Table 6 and the corresponding diagrams are presented in Figure 2. The $(V-J)_{0}$ interval in the second line of the table denotes the range of $(V-J)_{0}$ available for each cluster. 
Table 5. Colours and magnitudes for the clusters used in the calibration with 2MASS.

\begin{tabular}{|c|c|c|c|c|c|c|c|c|c|c|}
\hline \multirow{2}{*}{$\begin{array}{l}\text { M92 } \\
V\end{array}$} & \multirow[b]{2}{*}{$V-J$} & \multirow[b]{2}{*}{$J$} & \multirow[b]{2}{*}{$(V-J)_{0}$} & \multicolumn{3}{|c|}{ M13 } & \multirow[b]{2}{*}{$J$} & \multirow[b]{2}{*}{$(V-J)_{0}$} & \multirow[b]{2}{*}{$J_{0}$} & \\
\hline & & & & $J_{0}$ & $V$ & $V-J$ & & & & \\
\hline 21.994 & 1.883 & 20.111 & 1.827 & 20.089 & 22.039 & 2.011 & 20.028 & 1.966 & 20.011 & - \\
\hline 21.836 & 1.752 & 20.084 & 1.696 & 20.062 & 21.884 & 1.939 & 19.945 & 1.894 & 19.928 & - \\
\hline 21.688 & 1.684 & 20.004 & 1.628 & 19.982 & 21.740 & 1.883 & 19.857 & 1.838 & 19.840 & - \\
\hline 21.550 & 1.621 & 19.929 & 1.565 & 19.907 & 21.606 & 1.832 & 19.774 & 1.787 & 19.757 & - \\
\hline 21.420 & 1.570 & 19.850 & 1.514 & 19.828 & 21.460 & 1.766 & 19.694 & 1.721 & 19.677 & - \\
\hline 21.187 & 1.474 & 19.713 & 1.418 & 19.691 & 21.306 & 1.709 & 19.597 & 1.664 & 19.580 & - \\
\hline 21.064 & 1.433 & 19.631 & 1.377 & 19.609 & 21.173 & 1.653 & 19.520 & 1.608 & 19.503 & - \\
\hline 20.924 & 1.384 & 19.540 & 1.328 & 19.518 & 21.030 & 1.596 & 19.434 & 1.551 & 19.417 & - \\
\hline 20.792 & 1.347 & 19.445 & 1.291 & 19.423 & 20.891 & 1.541 & 19.350 & 1.496 & 19.333 & - \\
\hline 20.667 & 1.314 & 19.353 & 1.258 & 19.331 & 20.761 & 1.501 & 19.260 & 1.456 & 19.243 & - \\
\hline 20.537 & 1.280 & 19.257 & 1.224 & 19.235 & 20.640 & 1.455 & 19.185 & 1.410 & 19.168 & - \\
\hline 20.403 & 1.247 & 19.156 & 1.191 & 19.134 & 20.502 & 1.414 & 19.088 & 1.369 & 19.071 & - \\
\hline 20.273 & 1.216 & 19.057 & 1.160 & 19.035 & 20.372 & 1.368 & 19.004 & 1.323 & 18.987 & - \\
\hline 20.142 & 1.186 & 18.956 & 1.130 & 18.934 & 20.249 & 1.334 & 18.915 & 1.289 & 18.898 & - \\
\hline 20.000 & 1.155 & 18.845 & 1.099 & 18.823 & 20.112 & 1.299 & 18.813 & 1.254 & 18.796 & - \\
\hline 19.859 & 1.127 & 18.732 & 1.071 & 18.710 & 19.979 & 1.260 & 18.719 & 1.215 & 18.702 & - \\
\hline 19.716 & 1.099 & 18.617 & 1.043 & 18.595 & 19.839 & 1.222 & 18.619 & 1.175 & 18.602 & - \\
\hline 19.572 & 1.073 & 18.499 & 1.017 & 18.477 & 19.700 & 1.182 & 18.518 & 1.137 & 18.501 & - \\
\hline 19.422 & 1.047 & 18.375 & 0.991 & 18.353 & 19.560 & 1.146 & 18.414 & 1.101 & 18.397 & - \\
\hline 19.267 & 1.023 & 18.244 & 0.967 & 18.222 & 19.411 & 1.119 & 18.292 & 1.074 & 18.275 & - \\
\hline 19.105 & 1.000 & 18.105 & 0.944 & 18.083 & 19.263 & 1.095 & 18.168 & 1.050 & 18.151 & - \\
\hline 18.938 & 0.980 & 17.958 & 0.924 & 17.936 & 19.113 & 1.063 & 18.050 & 1.018 & 18.033 & - \\
\hline 18.780 & 0.965 & 17.815 & 0.909 & 17.793 & 18.962 & 1.042 & 17.920 & 0.997 & 17.903 & - \\
\hline \multirow[t]{2}{*}{18.617} & 0.953 & 17.664 & 0.897 & 17.642 & 18.800 & 1.024 & 17.776 & 0.979 & 17.759 & - \\
\hline & & & & & 18.637 & 1.008 & 17.629 & 0.963 & 17.612 & - \\
\hline NGC 6791 & & & & & NGC 2158 & & & & & \\
\hline$V$ & $V-J$ & $J$ & $(V-J)_{0}$ & $J_{0}$ & $(g-r)_{0}$ & $g_{0}$ & $(r-i)_{0}$ & $(g-J)_{0}$ & $(V-J)_{0}$ & $J_{0}$ \\
\hline $\begin{array}{l}9.817 \\
\end{array}$ & 2.062 & 17.755 & 1.725 & 17.625 & 0.123 & 15.744 & -0.018 & 0.652 & 0.569 & 15.092 \\
\hline 19.659 & 2.001 & 17.658 & 1.664 & 17.528 & 0.208 & 16.099 & 0.010 & 0.821 & 0.688 & 15.278 \\
\hline 19.515 & 1.944 & 17.571 & 1.607 & 17.441 & 0.299 & 16.947 & 0.061 & 1.033 & 0.846 & 15.914 \\
\hline 19.363 & 1.889 & 17.474 & 1.552 & 17.344 & 0.401 & 17.847 & 0.107 & 1.254 & 1.007 & 16.593 \\
\hline 19.215 & 1.840 & 17.375 & 1.503 & 17.245 & 0.498 & 18.480 & 0.149 & 1.462 & 1.158 & 17.018 \\
\hline 19.067 & 1.787 & 17.280 & 1.450 & 17.150 & 0.598 & 19.075 & 0.188 & 1.670 & 1.308 & 17.405 \\
\hline 18.924 & 1.738 & 17.186 & 1.401 & 17.056 & 0.693 & 19.371 & 0.217 & 1.856 & 1.438 & 17.515 \\
\hline 18.771 & 1.692 & 17.079 & 1.355 & 16.949 & 0.784 & 19.539 & 0.244 & 2.034 & 1.563 & 17.505 \\
\hline 18.633 & 1.656 & 16.977 & 1.319 & 16.847 & 0.878 & 19.749 & 0.293 & 2.247 & 1.720 & 17.502 \\
\hline 18.486 & 1.625 & 16.861 & 1.288 & 16.731 & - & - & - & - & - & - \\
\hline 18.331 & 1.595 & 16.736 & 1.258 & 16.606 & - & - & - & - & - & - \\
\hline 18.172 & 1.567 & 16.605 & 1.230 & 16.475 & - & - & - & - & - & - \\
\hline 18.011 & 1.548 & 16.463 & 1.211 & 16.333 & - & - & - & - & - & - \\
\hline 17.845 & 1.529 & 16.316 & 1.192 & 16.186 & - & - & - & - & - & - \\
\hline \multicolumn{11}{|l|}{47 Tuc } \\
\hline$V$ & $B-V$ & $R-I$ & $V_{0}$ & $(B-V)_{0}$ & $(R-I)_{0}$ & $(V-J)_{0}$ & $J_{0}$ & & & \\
\hline 19.700 & 0.662 & 0.487 & 19.018 & 0.442 & 0.355 & 0.901 & 18.117 & - & - & - \\
\hline 19.800 & 0.666 & 0.489 & 19.118 & 0.446 & 0.357 & 0.908 & 18.210 & - & - & - \\
\hline 19.900 & 0.669 & 0.493 & 19.218 & 0.449 & 0.361 & 0.915 & 18.303 & - & - & - \\
\hline 20.000 & 0.676 & 0.500 & 19.318 & 0.456 & 0.368 & 0.929 & 18.389 & - & - & - \\
\hline 20.100 & 0.681 & 0.506 & 19.418 & 0.461 & 0.374 & 0.939 & 18.479 & - & - & - \\
\hline 20.300 & 0.691 & 0.514 & 19.618 & 0.471 & 0.382 & 0.958 & 18.660 & - & - & - \\
\hline 20.400 & 0.700 & 0.518 & 19.718 & 0.480 & 0.386 & 0.974 & 18.744 & - & - & - \\
\hline 20.500 & 0.714 & 0.520 & 19.818 & 0.494 & 0.388 & 0.997 & 18.821 & - & - & - \\
\hline 20.600 & 0.722 & 0.522 & 19.918 & 0.502 & 0.390 & 1.010 & 18.908 & - & - & - \\
\hline 20.700 & 0.737 & 0.528 & 20.018 & 0.517 & 0.396 & 1.037 & 18.981 & - & - & - \\
\hline 20.800 & 0.752 & 0.534 & 20.118 & 0.532 & 0.402 & 1.063 & 19.055 & - & - & - \\
\hline 20.900 & 0.764 & 0.539 & 20.218 & 0.544 & 0.407 & 1.084 & 19.134 & - & - & - \\
\hline 21.000 & 0.777 & 0.545 & 20.318 & 0.557 & 0.413 & 1.107 & 19.211 & - & - & - \\
\hline 21.100 & 0.794 & 0.550 & 20.418 & 0.574 & 0.418 & 1.135 & 19.283 & - & - & - \\
\hline 21.200 & 0.809 & 0.561 & 20.518 & 0.589 & 0.429 & 1.164 & 19.354 & - & - & - \\
\hline 21.300 & 0.824 & 0.567 & 20.618 & 0.604 & 0.435 & 1.190 & 19.428 & - & - & - \\
\hline 21.400 & 0.838 & 0.571 & 20.718 & 0.618 & 0.439 & 1.214 & 19.504 & - & - & - \\
\hline 21.500 & 0.856 & 0.569 & 20.818 & 0.636 & 0.437 & 1.241 & 19.577 & - & - & - \\
\hline
\end{tabular}


Table 5. Continued.

\begin{tabular}{|c|c|c|c|c|c|c|c|c|c|c|}
\hline $\begin{array}{l}47 \text { Tuc } \\
V\end{array}$ & $B-V$ & $R-I$ & $V_{0}$ & $(B-V)_{0}$ & $(R-I)_{0}$ & $(V-J)_{0}$ & $J_{0}$ & & & \\
\hline 21.600 & 0.870 & 0.568 & 20.918 & 0.650 & 0.436 & 1.262 & 19.656 & - & - & - \\
\hline 21.700 & 0.887 & 0.569 & 21.018 & 0.667 & 0.437 & 1.289 & 19.729 & - & - & - \\
\hline 21.800 & 0.902 & 0.573 & 21.118 & 0.682 & 0.441 & 1.314 & 19.804 & - & - & - \\
\hline 21.900 & 0.917 & 0.577 & 21.218 & 0.697 & 0.445 & 1.339 & 19.879 & - & - & - \\
\hline 22.000 & 0.932 & 0.584 & 21.318 & 0.712 & 0.452 & 1.366 & 19.952 & - & - & - \\
\hline
\end{tabular}

Table 6. Numerical values of the coefficients $b_{i}(i=0,1,2,3,4,5)$.

\begin{tabular}{lrrrrr}
\hline \hline Cluster & M92 & M13 & 47 Tuc & NGC 2158 & NGC 6791 \\
$(V-J)_{0}$ interval & {$[0.90,1.83]$} & {$[0.96,1.96]$} & {$[0.90,1.37]$} & {$[0.57,1.31]$} & {$[1.19,1.72]$} \\
\hline$b_{5}$ & 13.498 & 11.536 & 152.580 & - & 74.861 \\
$b_{4}$ & -97.365 & -88.498 & -957.780 & - & -583.990 \\
$b_{3}$ & 278.950 & 269.890 & 2395.100 & -1.791 & 1819.900 \\
$b_{2}$ & -399.070 & -409.920 & -2982.400 & 3.395 & -2832.700 \\
$b_{1}$ & 288.640 & 312.580 & 1852.200 & 2.183 & 2204.800 \\
$b_{0}$ & -66.255 & -77.700 & -440.800 & 12.745 & -670.490 \\
\hline \hline
\end{tabular}

Table 7. $M_{g}$ absolute magnitudes estimated for a set of $(g-r)_{0}$ colours for six clusters used in the calibration with SDSS.

\begin{tabular}{|c|c|c|c|c|c|c|}
\hline$(g-r)_{0}$ & $\begin{array}{c}\text { M92 } \\
M_{g}\end{array}$ & $\begin{array}{c}\text { M13 } \\
M_{g}\end{array}$ & $\begin{array}{l}\text { M5 } \\
M_{g}\end{array}$ & $\begin{array}{c}\text { NGC } 2420 \\
M_{g}\end{array}$ & $\begin{array}{c}\text { M67 } \\
M_{g}\end{array}$ & $\begin{array}{c}\text { NGC } 6791 \\
M_{g} \\
\end{array}$ \\
\hline 0.20 & 4.199 & - & - & 3.321 & - & - \\
\hline 0.25 & 4.972 & 4.603 & 4.475 & 3.858 & - & - \\
\hline 0.30 & 5.460 & 5.275 & 5.148 & 4.320 & - & - \\
\hline 0.35 & 5.864 & 5.746 & 5.611 & 4.723 & 4.442 & - \\
\hline 0.40 & 6.178 & 6.107 & 5.965 & 5.079 & 4.883 & - \\
\hline 0.45 & 6.462 & 6.417 & 6.273 & 5.399 & 5.261 & - \\
\hline 0.50 & - & 6.704 & 6.563 & 5.691 & 5.593 & - \\
\hline 0.55 & - & 6.978 & 6.840 & 5.961 & 5.890 & 5.024 \\
\hline 0.60 & - & 7.240 & 7.100 & 6.213 & 6.160 & 5.451 \\
\hline 0.65 & - & 7.485 & 7.333 & 6.450 & 6.410 & 5.779 \\
\hline 0.70 & - & 7.717 & 7.537 & 6.675 & 6.644 & 6.048 \\
\hline 0.75 & - & 7.953 & 7.728 & 6.887 & 6.865 & 6.283 \\
\hline 0.80 & - & - & 7.945 & 7.089 & 7.073 & 6.503 \\
\hline 0.85 & - & - & - & 7.281 & 7.271 & 6.717 \\
\hline 0.90 & - & - & - & 7.464 & 7.458 & 6.927 \\
\hline 0.95 & - & - & - & 7.639 & 7.636 & 7.136 \\
\hline 1.00 & - & - & - & 7.808 & 7.807 & 7.340 \\
\hline 1.05 & - & - & - & 7.976 & 7.975 & 7.539 \\
\hline 1.10 & - & - & - & 8.146 & 8.145 & 7.734 \\
\hline 1.15 & - & - & - & 8.327 & 8.324 & 7.930 \\
\hline 1.20 & - & - & - & 8.526 & 8.523 & 8.139 \\
\hline 1.25 & - & - & - & 8.755 & 8.757 & 8.381 \\
\hline 1.30 & - & - & - & 9.028 & 9.043 & - \\
\hline 1.35 & - & - & - & 9.363 & 9.402 & - \\
\hline
\end{tabular}

\section{THE PROCEDURE}

\subsection{Absolute magnitude as a function of metallicity}

We adopted the procedure used in our previous papers (Karaali et al. 2013a, 2013b) which consists of calibration of an absolute magnitude as a function of metallicity. We calibrated the $M_{g}$ and $M_{J}$ absolute magnitudes in terms of metallicity for a given $(g-r)_{0}$ and $(V-J)_{0}$ colour, respectively.

\subsubsection{Calibration of $M_{g}$ in terms of metallicity}

We estimated the $M_{g}$ absolute magnitude for the $(g-r)_{0}$ colours given in Table 7 for the cluster sample in Table 1 by combining the $g_{0}$ apparent magnitude evaluated by 


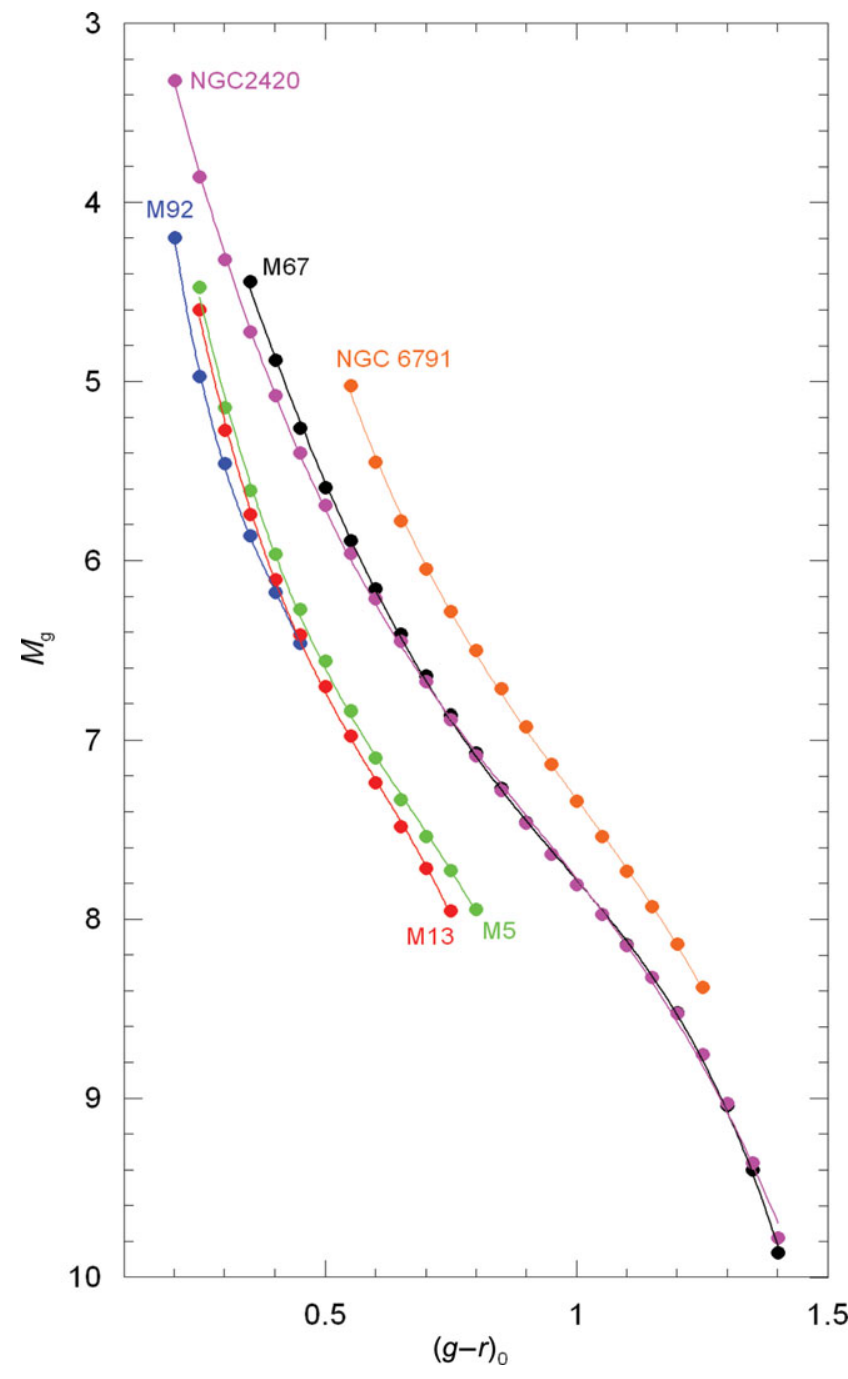

Figure 3. $M_{g} \times(g-r)_{0}$ colour-magnitude diagram for six clusters used for the absolute magnitude calibration with SDSS.

Equation (1) and the true distance modulus $\left(\mu_{0}\right)$ of the cluster in question, i.e.,

$$
M_{g}=g_{0}-\mu_{0}
$$

The $M_{g} \times(g-r)_{0}$ absolute magnitude-colour diagrams are plotted in Figure 3. The absolute magnitudes of the clusters M13 $([\mathrm{Fe} / \mathrm{H}]=-1.41 \mathrm{dex})$ and M5 $([\mathrm{Fe} / \mathrm{H}]=-1.26$ dex) are close to each other for a given colour. Similar case is valid for the clusters NGC $2420([\mathrm{Fe} / \mathrm{H}]=-0.37 \mathrm{dex})$ and M67 $([\mathrm{Fe} / \mathrm{H}]=-0.04 \mathrm{dex})$. However, we used both couples to obtain a better absolute magnitude calibration.

In this phase, $M_{g}$ absolute magnitudes can be fitted to the corresponding $[\mathrm{Fe} / \mathrm{H}]$ metal abundance for a given $(g-r)_{0}$ colour index and obtain the required calibration. This procedure is executed for the colour indices $(g-r)_{0}=0.25$, $0.40,0.65,0.80,0.95$, and 1.20 (Table 8 and Figure 4). The absolute magnitudes in all colour indices are fitted to a second-degree polynomial with (squared) correlation co-
Table 8. $M_{g}$ absolute magnitudes and $[\mathrm{Fe} / \mathrm{H}]$ metallicities for six $(g-r)_{0}$ intervals.

\begin{tabular}{lccccc}
\hline \hline $\begin{array}{l}(g-r)_{0} \\
(\mathrm{mag})\end{array}$ & $\begin{array}{c}{[\mathrm{Fe} / \mathrm{H}]} \\
(\mathrm{dex})\end{array}$ & $\begin{array}{c}M_{g} \\
(\mathrm{mag})\end{array}$ & $\begin{array}{c}(g-r)_{0} \\
(\mathrm{mag})\end{array}$ & $\begin{array}{c}{[\mathrm{Fe} / \mathrm{H}]} \\
(\mathrm{dex})\end{array}$ & $\begin{array}{c}M_{g} \\
(\mathrm{mag})\end{array}$ \\
\hline 0.25 & -2.15 & 4.972 & 0.80 & -1.26 & 7.945 \\
& -1.41 & 4.603 & & -0.37 & 7.089 \\
& -1.26 & 4.475 & & -0.04 & 7.073 \\
& -0.37 & 3.858 & & 0.37 & 6.503 \\
0.40 & -2.15 & 6.178 & 0.95 & -0.37 & 7.639 \\
& -1.41 & 6.107 & & -0.04 & 7.636 \\
& -1.26 & 5.965 & & 0.37 & 7.136 \\
& -0.37 & 5.079 & 1.20 & -0.37 & 8.526 \\
& -0.04 & 4.883 & & -0.04 & 8.523 \\
0.65 & -1.41 & 7.485 & & 0.37 & 8.139 \\
& -1.26 & 7.333 & - & - & - \\
& -0.37 & 6.450 & - & - & - \\
& -0.04 & 6.410 & - & - & - \\
& 0.37 & 5.779 & - & - & - \\
\hline \hline
\end{tabular}

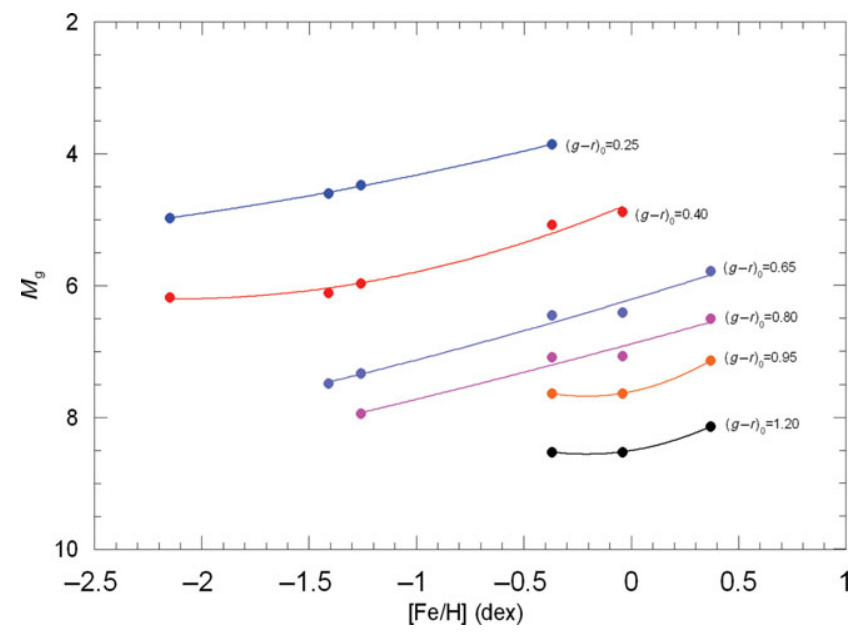

Figure 4. Calibration of the absolute magnitude $M_{g}$ as a function of metallicity $[\mathrm{Fe} / \mathrm{H}]$ for six colour indices.

efficients between $R^{2}=0.962$ and $R^{2}=0.999$, where the first one corresponds to the absolute magnitudes for the colour index $(g-r)_{0}=0.80 \mathrm{mag}$ (but see the following paragraph). A third-degree polynomial (Figure 5) increases the correlation coefficient to $R^{2}=1$. However, it causes a flat distribution in the metallicity interval $-0.90 \leq[\mathrm{Fe} / \mathrm{H}] \leq$ -0.40 dex, resulting almost a constant absolute magnitude in this metallicity interval which contradicts the trends of absolute magnitudes for small colour indices, such as $(g-r)_{0}=0.35 \mathrm{mag}$, where the correlation coefficient is high, $R^{2}=0.991$. We shall see in Section 3.2 that the application of the third-degree polynomial in question causes larger residuals than the ones for a quadratic polynomial, $\Delta M=-0.360$ and $0.067 \mathrm{mag}$, respectively.

The number of clusters in the absolute magnitude calibration for the colour indices $0.82 \leq(g-r)_{0} \leq 1.25 \mathrm{mag}$ is only three, i.e., NGC 2420, M67, and NGC 6791 with metallicities $[\mathrm{Fe} / \mathrm{H}]=-0.37,[\mathrm{Fe} / \mathrm{H}]=-0.04$, and $[\mathrm{Fe} / \mathrm{H}]=$ 

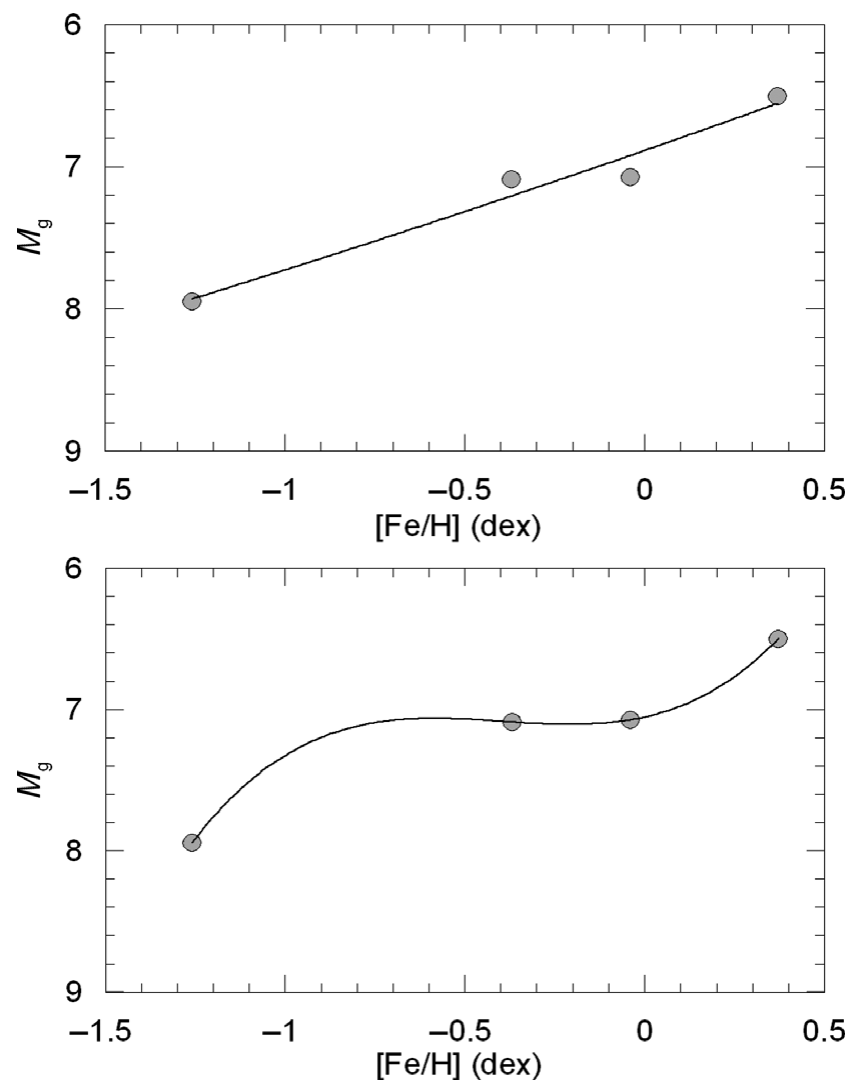

Figure 5. Comparison the trends of the absolute magnitude calibrations with polynomial degrees $n=2$ (upper panel) and $n=3$ (lower panel) for the colour index $(g-r)_{0}=0.80 \mathrm{mag}$.

0.37 dex, respectively. Additionally, the absolute magnitudes of the clusters NGC 2420 and M67 are close to each other. If we fit a quadratic polynomial to three metallicity and absolute magnitude couples, the segment corresponding to the interval $-0.37 \leq[\mathrm{Fe} / \mathrm{H}] \leq-0.04$ dex will perform a concave shape resulting in (estimated) absolute magnitudes larger than the ones for the cluster NGC 2420 which is opposite to the sense of the argument used in our work, i.e., the absolute magnitude of a dwarf with a given metallicity and colour should be brighter than one relatively more metal-poor. Hence, we fitted a linear equation to three metallicity and absolute magnitude couples in question (Figure 6b).

The procedure can be applied to any $(g-r)_{0}$ colour interval for which the sample clusters are defined. The $(g-r)_{0}$ domains of the clusters are different. Hence, we adopted this interval in our study as $0.25 \leq(g-r)_{0} \leq 1.25$ mag where at least three clusters are defined, and we evaluated $M_{g}$ absolute magnitudes for each colour. Then, we combined them with the corresponding $[\mathrm{Fe} / \mathrm{H}]$ metallicities and obtained the final calibrations. The general form of the equation for the calibrations is as follows:

$$
M_{g}=c_{0}+c_{1} X+c_{2} X^{2},
$$

where $X=[\mathrm{Fe} / \mathrm{H}]$. The absolute magnitudes estimated via Equation (7) for $101(g-r)_{0}$ colour indices and the corre-

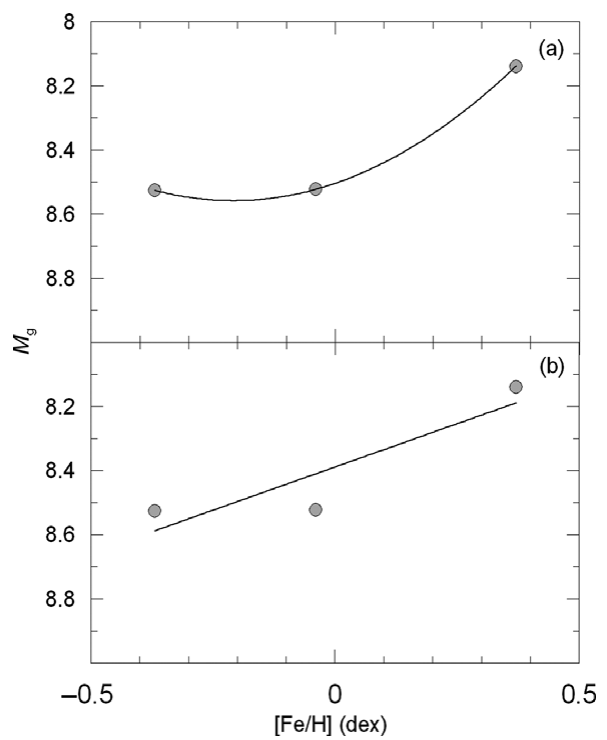

Figure 6. Two fittings to the metallicities and absolute magnitudes for the colour index $(g-r)_{0}=1.20$ dex. (a) a quadratic polynomial to three couples and (b) a linear polynomial to three couples.

sponding $c_{i}(i=0,1,2)$ coefficients are given in Table 9 . The coefficients $c_{1}$ and $c_{0}$ for the linear equation

$$
M_{g}=c_{0}+c_{1} X,
$$

which are valid for the colour interval $0.82 \leq(g-r)_{0} \leq$ 1.25 are also tabulated in Table 9. The diagrams for the calibrations are not given in the paper because of space constraints. One can use any dataset taken from Table 9 depending on the desire for accuracy, and apply it to stars whose iron abundances are available.

\subsubsection{Calibration of $M_{J}$ in terms of metallicity}

We estimated the $M_{J}$ absolute magnitude for the $(V-J)_{0}$ colours given in Table 10 for the cluster sample in Table 4 by combining the $J_{0}$ apparent magnitude evaluated by Equation (6) and the true distance modulus $\left(\mu_{0}\right)$ of the cluster in question, i.e.,

$$
M_{J}=J_{0}-\mu_{0}
$$

The absolute magnitudes versus $(V-J)_{0}$ colours are plotted in Figure 7. We fitted the $M_{J}$ absolute magnitudes to the corresponding $[\mathrm{Fe} / \mathrm{H}]$ metallicity for the following $(\mathrm{V}-\mathrm{J})_{0}$ colour indices and obtained the required calibrations just for the exhibition of the procedure: $(V-J)_{0}=1.00,1.15$, 1.30, 1.50, and 1.70. The results are given in Table 11 and Figure 8 . The absolute magnitudes in all colour indices are fitted to a quadratic polynomial with (squared) correlation coefficients $\left(R^{2}\right)$ between 0.9667 and 1 , where the first one corresponds to the absolute magnitudes for the colour index $(V-J)_{0}=1.15$ mag. 
Table 9. Absolute magnitudes estimated for six Galactic clusters and the numerical values of $c_{i}(i=0,1,2)$ coefficients in Equations (8) and (9).

\begin{tabular}{|c|c|c|c|c|c|c|c|c|c|c|c|c|c|c|c|c|c|c|c|c|c|c|c|}
\hline & M92 & M13 & M5 & $\begin{array}{l}\text { NGC } \\
2420 \\
\end{array}$ & M67 & $\begin{array}{l}\text { NGC } \\
6791 \\
\end{array}$ & & & & & & & M92 & M13 & M5 & $\begin{array}{l}\text { NGC } \\
2420 \\
\end{array}$ & M67 & $\begin{array}{l}\text { NGC } \\
6791 \\
\end{array}$ & (cont.) & & & & \\
\hline$(g-r)_{0}$ & $M_{g}$ & $M_{g}$ & $M_{g}$ & $M_{g}$ & $M_{g}$ & $M_{g}$ & $c_{2}$ & $c_{1}$ & $c_{0}$ & $R^{2}$ & $\begin{array}{l}{[\mathrm{Fe} / \mathrm{H}]} \\
\text { interval }\end{array}$ & $(g-r)_{0}$ & $M_{g}$ & $M_{g}$ & $M_{g}$ & $M_{g}$ & $M_{g}$ & $M_{g}$ & $c_{2}$ & $c_{1}$ & $c_{0}$ & $R^{2}$ & $\begin{array}{l}{[\mathrm{Fe} / \mathrm{H}]} \\
\text { interval }\end{array}$ \\
\hline 25 & 4.972 & 4.603 & 4.475 & 3.858 & - & - & -0.0977 & -0.8738 & 3.5469 & 0.9990 & {$[-2.15,-0.37]$} & 0.76 & - & 8.004 & 7.767 & 6.929 & 6.908 & 6.328 & 0.0094 & -0.8681 & 16.7093 & 0.9754 & {$[-1.41,0.3$} \\
\hline 5 & 5.081 & 4.759 & 4.633 & 3.956 & - & - & 0.1663 & 527 & 5880 & 0.999 & {$[-2.15,-0$.} & & - & 8.057 & 7.808 & 6.970 & 6.950 & 6.372 & 82 & 609 & 96.7507 & & \\
\hline 27 & 5.182 & 4.904 & 4.778 & 4.051 & - & - & -0.2255 & -1.2054 & 3.6346 & 0.9991 & {$[-2.15,-0.37]$} & 0.78 & - & 8.113 & 7.851 & 7.010 & 6.991 & 6.416 & 0.0285 & -0.8538 & 86.7915 & 0.97 & {$[-1.41, \mathrm{C}$} \\
\hline .28 & 5.278 & 5.037 & 4.912 & 4.143 & - & - & -0.2754 & -1.3335 & 3.6863 & 0.9991 & {$[-2.15,-0.37]$} & 0.79 & - & - & 7.896 & 7.050 & 7.033 & 6.460 & -0.0492 & -0.8850 & 06.8449 & 0.96 & {$[-1.26,0.37]$} \\
\hline .29 & 5.371 & 5.161 & 5.035 & 4.233 & - & - & -0.3166 & -1.4388 & 3.7426 & 0.9991 & {$[-2.15,-0.37]$} & 0.80 & - & - & 7.945 & 7.089 & 7.073 & 6.503 & -0.0403 & -0.8803 & 36.8854 & 0.9625 & {$[-1.26,0.37]$} \\
\hline 0.30 & 5.460 & 5.275 & 5.148 & 4.320 & - & - & -0.3498 & -1.5235 & 3.8030 & 0.9990 & {$[-2.15,-0.37]$} & 0.81 & - & - & 7.998 & 7.129 & 7.114 & 6.546 & -0.0284 & -0.8755 & 56.9252 & 0.9628 & {$[-1.26,0.37]$} \\
\hline 31 & 5.546 & 5.382 & 5.254 & 4.405 & - & - & -0.3758 & -1.5901 & 3.8667 & 0.9989 & {$[-2.15,-0.37]$} & 0.82 & - & - & - & 7.167 & 7.154 & 6.589 & - & -0.8054 & 46.9593 & 0.8181 & {$[-0.37,0.37]$} \\
\hline 0.32 & 5.630 & 5.482 & 5.352 & 4.488 & - & - & -0.3958 & -1.6412 & 3.9330 & 0.9989 & {$[-2.15,-0.37]$} & 0.83 & - & - & - & 7.206 & 7.193 & 6.632 & - & -0.7995 & 56.9995 & 0.8169 & {$[-0.37,0.37]$} \\
\hline 0.33 & 5.711 & 5.575 & 5.443 & 4.568 & - & - & -0.4108 & -1.6794 & 4.0013 & 0.9988 & {$[-2.15,-0.37]$} & 0.84 & - & - & - & 7.244 & 7.232 & 6.674 & - & -0.7932 & 27.0395 & 0.8157 & {$[-0.37,0.37]$} \\
\hline 0.34 & 5.789 & 5.663 & 5.530 & 4.646 & - & - & -0.4220 & -1.7075 & 4.0707 & 0.9987 & {$[-2.15,-0.37]$} & 0.85 & - & - & - & 7.281 & 7.271 & 6.717 & - & -0.7866 & 67.0791 & 0.8145 & {$[-0.37,0.37]$} \\
\hline 0.35 & 5.864 & 5.746 & 5.611 & 4.723 & 4.442 & - & -0.3313 & -1.4360 & 4.3272 & 0.9913 & {$[-2.15,-0.04]$} & 0.86 & - & - & - & 7.319 & 7.309 & 6.759 & - & -0.7796 & 67.1185 & 0.8134 & {$[-0.37,0.37]$} \\
\hline 0.36 & 5.934 & 5.824 & 5.688 & 4.797 & 4.536 & - & -0.3281 & -1.4217 & 4.4159 & 0.9893 & {$[-2.15,-0.04]$} & 0.87 & - & - & - & 7.355 & 7.347 & 6.801 & - & -0.7 & 27.1575 & 0.8124 & {$[-0.37,0.37]$} \\
\hline 0.37 & 6.001 & 5.900 & 5.761 & 4.870 & 4.627 & - & -0.325 & -1.4070 & 4.5020 & 0.9873 & {$[-2.15,-0.04]$} & 0.88 & - & - & - & 7.392 & 7.384 & 6.843 & - & -0.7 & 47.1963 & 0.8113 & {$[-0.37,0.37]$} \\
\hline 0.38 & 6.064 & 5.971 & 5.832 & 4.941 & 4.715 & - & -0.3226 & -1.3927 & 4.5857 & 0.9852 & {$[-2.15,-0.04]$} & $0.8 \mathrm{~s}$ & - & - & - & 7.428 & 7.421 & 6.885 & - & -0 & 27.2349 & & {$[-0.37,0.37]$} \\
\hline 0.39 & 6.123 & 6.040 & 5.900 & 5.011 & 4.800 & - & -0.3211 & -1.3796 & 4.6670 & 0.9829 & {$[-2.15,-0.04]$} & 0.90 & - & - & - & 7.464 & 7.458 & 6.927 & - & -0.7 & 77.2732 & 0.8094 & {$[-0.37,0.37]$} \\
\hline 0.40 & 6.178 & 6.107 & 5.965 & 5.079 & 4.883 & - & -0.3203 & -1.3677 & 4.7459 & 0.9806 & {$[-2.15,-0.04]$} & 0.91 & - & - & - & 7.499 & 7.494 & 6.969 & - & -0.7389 & 97.3112 & 0.8085 & {$[-0.37,0.37]$} \\
\hline 0.41 & 6.231 & 6.172 & 6.029 & 5.146 & 4.963 & - & -0.3201 & -1.3570 & 4.8225 & 0.9782 & {$[-2.15,-0.04]$} & 0.92 & - & - & - & 7.535 & 7.530 & 7.011 & - & -0.7298 & 87.3490 & 0.8077 & {$[-0.37,0.37]$} \\
\hline 0.42 & 6.283 & 6.235 & 6.092 & 5.211 & 5.040 & - & -0.3198 & -1.3465 & 4.8972 & 0.9758 & {$[-2.15,-0.04]$} & 0.93 & - & - & - & 7.570 & 7.566 & 7.053 & - & -0.7205 & 57.3865 & 0.8069 & {$[-0.37,0.37]$} \\
\hline 0.43 & 6.337 & 6.297 & 6.153 & 5.275 & 5.116 & - & -0.3183 & -1.3349 & 4.9702 & 0.9736 & {$[-2.15,-0.04]$} & 0.94 & - & - & - & 7.604 & .7 .601 & 7.094 & - & & 07.4238 & 0.8062 & {$[-0.37,0.37]$} \\
\hline 0.44 & 6.396 & 6.357 & 6.214 & 5.337 & 5.190 & - & -0.3142 & -1.3201 & 5.0417 & 0.9717 & {$[-2.15,-0.04]$} & 0.95 & - & - & - & 7.639 & 7.636 & 7.136 & - & -0.7014 & 47.4609 & 0.8055 & {$[-0.37,0.37]$} \\
\hline 0.45 & 6.462 & 6.417 & 6.273 & 5.399 & 5.261 & - & -0.3054 & -1.2994 & 5.1124 & 0.9706 & {$[-2.15,-0.04]$} & 0.96 & - & - & - & 7.673 & 7.671 & 7.177 & - & -0.6916 & 67.4977 & 0.8049 & {$[-0.37,0.37]$} \\
\hline 0.46 & 6.541 & 6.475 & 6.332 & 5.459 & 5.331 & - & -0.2895 & -1.2693 & 5.1827 & 0.9704 & {$[-2.15,-0.04]$} & 0.97 & - & - & - & 7.707 & 7.705 & 7.218 & - & -0.6818 & 87.5343 & 0.8044 & {$[-0.37,0.37]$} \\
\hline 0.47 & - & 6.533 & 6.390 & 5.519 & 5.399 & - & 0.3926 & -0.2839 & 5.3772 & 0.9980 & {$[-1.41,-0.04]$} & 0.98 & - & - & - & 7.741 & 7.739 & 7.259 & - & -0.6721 & 17.5708 & 0.8039 & {$[-0.37,0.37]$} \\
\hline 0.48 & - & 6.591 & 6.448 & 5.577 & 5.465 & - & 0.4071 & -0.2573 & 5.4441 & 0.9978 & {$[-1.41,-0.04]$} & 0.99 & - & - & - & 7.775 & 7.773 & 7.300 & - & -0.6624 & 47.6070 & 0.8035 & {$[-0.37,0.37]$} \\
\hline 0.49 & - & 6.647 & 6.506 & 5.634 & 5.530 & - & 0.4207 & -0.2329 & 5.5093 & 0.9976 & {$[-1.41,-0.04]$} & 1.00 & - & - & - & 7.808 & 7.807 & 7.340 & - & -0.6529 & 97.6431 & 0.8032 & {$[-0.37,0.37]$} \\
\hline
\end{tabular}


Table 9. Continued.

\begin{tabular}{|c|c|c|c|c|c|c|c|c|c|c|c|c|c|c|c|c|c|c|c|c|c|c|c|}
\hline & M92 & M13 & M5 & $\begin{array}{l}\text { NGC } \\
2420\end{array}$ & M67 & $\begin{array}{l}\text { NGC } \\
6791\end{array}$ & & & & & & & M92 & M13 & M5 & $\begin{array}{l}\text { NGC } \\
2420\end{array}$ & M67 & $\begin{array}{l}\text { NGC } \\
6791\end{array}$ & (cont.) & & & & \\
\hline$(g-r)_{0}$ & $M_{g}$ & $M_{g}$ & $M_{g}$ & $M_{g}$ & $M_{g}$ & $M_{g}$ & $c_{2}$ & $c_{1}$ & $c_{0}$ & $R^{2}$ & $\begin{array}{l}{[\mathrm{Fe} / \mathrm{H}]} \\
\text { interval }\end{array}$ & $(g-r)_{0}$ & $M_{g}$ & $M_{g}$ & $M_{g}$ & $M_{g}$ & $M_{g}$ & $M_{g}$ & $c_{2}$ & $c_{1}$ & $c_{0}$ & $R^{2}$ & $\begin{array}{l}{[\mathrm{Fe} / \mathrm{H}]} \\
\text { interval }\end{array}$ \\
\hline 0.50 & - & 6.704 & 6.563 & 5.691 & 5.593 & - & 0.4332 & -0.2106 & 5.5730 & 0.9974 & {$[-1.41,-0.04]$} & 1.01 & - & - & - & 7.842 & 7.841 & 7.380 & - & -0.6435 & 7.6791 & 0.8030 & {$[-0.37,0.37]$} \\
\hline 0.51 & - & 6.760 & 6.619 & 5.747 & 5.655 & - & 0.4450 & -0.1902 & 5.6353 & 0.9972 & {$[-1.41,-0.04]$} & 1.02 & - & - & - & 7.875 & 7.875 & 7.420 & - & -0.6344 & 7.7149 & 0.8029 & {$[-0.37,0.37]$} \\
\hline 0.52 & - & 6.815 & 6.675 & 5.801 & 5.715 & - & 0.4560 & -0.1715 & 5.6961 & 0.9970 & {$[-1.41,-0.04]$} & 1.03 & - & - & - & 7.909 & 7.908 & 7.460 & - & -0.6255 & 7.7506 & 0.8028 & {$[-0.37,0.37]$} \\
\hline 0.53 & - & 6.870 & 6.731 & 5.855 & 5.775 & - & 0.4664 & -0.1542 & 5.7557 & 0.9968 & {$[-1.41,-0.04]$} & 1.04 & - & - & - & 7.942 & 7.942 & 7.500 & - & -0.6170 & 7.7862 & 0.8029 & {$[-0.37,0.37]$} \\
\hline 0.54 & - & 6.924 & 6.786 & 5.908 & 5.833 & - & 0.4763 & -0.1382 & 5.8141 & 0.9966 & {$[-1.41,-0.04]$} & 1.05 & - & - & - & 7.976 & 7.975 & 7.539 & - & -0.6089 & 7.8218 & 0.8030 & {$[-0.37,0.37]$} \\
\hline 0.55 & - & 6.978 & 6.840 & 5.961 & 5.890 & 5.024 & -0.3039 & -1.3558 & 5.6378 & 0.9745 & {$[-1.41,0.37]$} & 1.06 & - & - & - & 8.009 & 8.009 & 7.578 & - & -0.6011 & 7.8574 & 0.8031 & {$[-0.37,0.37]$} \\
\hline 0.56 & - & 7.032 & 6.894 & 6.013 & 5.946 & 5.120 & -0.2652 & -1.2953 & 5.7028 & 0.9753 & {$[-1.41,0.37]$} & 1.07 & - & - & - & 8.043 & 8.042 & 7.617 & - & -0.5938 & 7.8930 & 0.8034 & {$[-0.37,0.37]$} \\
\hline 0.57 & - & 7.085 & 6.947 & 6.064 & 6.001 & 5.209 & -0.2306 & -1.2414 & 5.7658 & 0.9760 & {$[-1.41,0.37]$} & 1.08 & - & - & - & 8.077 & 8.076 & 7.656 & - & -0.5870 & 7.9287 & 0.8037 & {$[-0.37,0.37]$} \\
\hline 0.58 & - & 7.137 & 6.999 & 6.114 & 6.055 & 5.294 & -0.1999 & -1.1936 & 5.8267 & 0.9767 & {$[-1.41,0.37]$} & 1.09 & - & - & - & 8.112 & 8.110 & 7.695 & - & -0.5807 & 7.9645 & 0.8041 & {$[-0.37,0.37]$} \\
\hline 0.59 & - & 7.189 & 7.050 & 6.164 & 6.108 & 5.375 & -0.1727 & -1.1513 & 5.8859 & 0.9772 & {$[-1.41,0.37]$} & 1.10 & - & - & - & 8.146 & 8.145 & 7.734 & - & -0.5748 & 8.0006 & 0.8045 & {$[-0.37,0.37]$} \\
\hline 0.60 & - & 7.240 & 7.100 & 6.213 & 6.160 & 5.451 & -0.1487 & -1.1140 & 5.9433 & 0.9776 & {$[-1.41,0.37]$} & 1.11 & - & - & - & 8.181 & 8.179 & 7.773 & - & -0.5695 & 8.0369 & 0.8049 & {$[-0.37,0.37]$} \\
\hline 0.61 & - & 7.290 & 7.149 & 6.261 & 6.212 & 5.523 & -0.1276 & -1.0811 & 5.9992 & 0.9780 & {$[-1.41,0.37]$} & 1.12 & - & - & - & 8.217 & 8.215 & 7.812 & - & -0.5646 & 8.0735 & 0.8054 & {$[-0.37,0.37]$} \\
\hline 0.62 & - & 7.340 & 7.197 & 6.309 & 6.262 & 5.591 & -0.1092 & -1.0523 & 6.0537 & 0.9783 & {$[-1.41,0.37]$} & 1.13 & - & - & - & 8.253 & 8.250 & 7.851 & - & -0.5602 & 8.1105 & 0.8058 & {$[-0.37,0.37]$} \\
\hline 0.63 & - & 7.389 & 7.243 & 6.357 & 6.312 & 5.657 & -0.0932 & -1.0270 & 6.1068 & 0.9785 & {$[-1.41,0.37]$} & 1.14 & - & - & - & 8.289 & 8.287 & 7.890 & - & -0.5563 & 8.1480 & 0.8061 & {$[-0.37,0.37]$} \\
\hline 0.64 & - & 7.437 & 7.289 & 6.404 & 6.362 & 5.719 & -0.0794 & -1.0048 & 6.1586 & 0.9786 & {$[-1.41,0.37]$} & 1.15 & - & - & - & 8.327 & 8.324 & 7.930 & - & -0.5528 & 8.1861 & 0.8064 & {$[-0.37,0.37]$} \\
\hline 0.65 & - & 7.485 & 7.333 & 6.450 & 6.410 & 5.779 & -0.0674 & -0.9854 & 6.2093 & 0.9786 & {$[-1.41,0.37]$} & 1.16 & - & - & - & 8.365 & 8.362 & 7.970 & - & -0.5496 & 8.2249 & 0.8066 & {$[-0.37,0.37]$} \\
\hline 0.66 & - & 7.532 & 7.376 & 6.496 & 6.458 & 5.836 & -0.0571 & -0.9684 & 6.2589 & 0.9786 & {$[-1.41,0.37]$} & 1.17 & - & - & - & 8.403 & 8.401 & 8.011 & - & -0.5468 & 8.2644 & 0.8067 & {$[-0.37,0.37]$} \\
\hline 0.67 & - & 7.579 & 7.418 & 6.541 & 6.506 & 5.892 & -0.0482 & -0.9533 & 6.3075 & 0.9786 & {$[-1.41,0.37]$} & 1.18 & - & - & - & 8.443 & 8.440 & 8.053 & - & -0.5441 & 8.3048 & 0.8066 & {$[-0.37,0.37]$} \\
\hline 0.68 & - & 7.625 & 7.459 & 6.586 & 6.552 & 5.945 & -0.0405 & -0.9401 & 6.3552 & 0.9784 & {$[-1.41,0.37]$} & 1. & - & - & - & 8.484 & 8.481 & 8.095 & - & -0.5416 & 8.3463 & 0.8063 & {$[-0.37,0.37]$} \\
\hline 0.69 & - & 7.671 & 7.498 & 6.631 & 6.599 & 5.997 & -0.0336 & -0.9283 & 6.4020 & 0.9782 & {$[-1.41,0.37]$} & 1.20 & - & - & - & 8.526 & 8.523 & 8.139 & - & -0.5391 & 8.3888 & 0.8058 & {$[-0.37,0.37]$} \\
\hline 0.70 & - & 7.717 & 7.537 & 6.675 & 6.644 & 6.048 & -0.0275 & -0.9176 & 6.4480 & 0.9780 & {$[-1.41,0.37]$} & 1.21 & - & - & - & 8.569 & 8.567 & 8.184 & - & -0.5365 & 8.4327 & 0.8050 & {$[-0.37,0.37]$} \\
\hline 0.71 & - & 7.763 & 7.576 & 6.718 & 6.689 & 6.097 & -0.0218 & -0.9080 & 6.4932 & 0.9777 & {$[-1.41,0.37]$} & 1.22 & - & - & - & 8.613 & 8.612 & 8.230 & - & -0.5337 & 8.4780 & 0.8038 & {$[-0.37,0.37]$} \\
\hline 0.72 & - & 7.809 & 7.613 & 6.761 & 6.734 & 6.145 & -0.0163 & -0.8991 & 6.5378 & 0.9773 & {$[-1.41,0.37]$} & 1.23 & - & - & - & 8.659 & 8.659 & 8.278 & - & -0.5305 & 8.5248 & 0.8023 & {$[-0.37,0.37]$} \\
\hline 0.73 & - & 7.856 & 7.651 & 6.804 & 6.778 & 6.192 & -0.0107 & -0.8908 & 6.5816 & 0.9769 & {$[-1.41,0.37]$} & 1.24 & - & - & - & 8.706 & 8.707 & 8.328 & - & -0.5267 & 8.5734 & 0.8003 & {$[-0.37,0.37]$} \\
\hline 0.74 & - & 7.904 & 7.689 & 6.846 & 6.822 & 6.238 & -0.0047 & -0.8830 & 6.6248 & 0.9764 & {$[-1.41,0.37]$} & 1.25 & - & - & - & 8.755 & 8.757 & 8.381 & - & -0.5221 & 8.6240 & 0.7977 & {$[-0.37,0.37]$} \\
\hline 0.75 & - & 7.953 & 7.728 & 6.887 & 6.865 & 6.283 & -0.0019 & -0.8755 & 6.6674 & 0.9759 & {$[-1.41,0.37]$} & - & - & - & - & - & - & - & - & - & - & - & - \\
\hline
\end{tabular}


Table 10. $M_{J}$ absolute magnitudes estimated for a set of ( $V-$ $J)_{0}$ colours for five clusters used for the calibration with 2MASS. The absolute magnitudes with boldface are not considered in the calibration (see text).

\begin{tabular}{|c|c|c|c|c|c|}
\hline $\begin{array}{l}\text { Cluster } \\
(V-J)_{0} \\
(\mathrm{mag})\end{array}$ & $\begin{array}{c}\text { M92 } \\
M_{J} \\
\text { (mag) }\end{array}$ & $\begin{array}{c}\mathrm{M} 13 \\
M_{J} \\
\text { (mag) }\end{array}$ & $\begin{array}{c}47 \text { Tuc } \\
M_{J} \\
(\mathrm{mag})\end{array}$ & $\begin{array}{c}\text { NGC } 2158 \\
M_{J} \\
(\mathrm{mag})\end{array}$ & $\begin{array}{c}\text { NGC } 6791 \\
M_{J} \\
(\mathrm{mag})\end{array}$ \\
\hline 0.90 & 3.00 & - & 2.76 & 2.77 & - \\
\hline 0.95 & 3.38 & 3.16 & 3.22 & 2.97 & - \\
\hline 1.00 & 3.68 & 3.51 & 3.50 & 3.15 & - \\
\hline 1.05 & 3.92 & 3.78 & 3.69 & 3.33 & - \\
\hline 1.10 & 4.12 & 3.99 & 3.84 & 3.49 & - \\
\hline 1.15 & 4.30 & 4.16 & 3.98 & 3.64 & - \\
\hline 1.20 & 4.45 & 4.30 & 4.13 & 3.78 & 3.19 \\
\hline 1.25 & 4.59 & 4.42 & 4.29 & 3.90 & 3.51 \\
\hline 1.30 & 4.71 & 4.53 & 4.44 & 4.00 & 3.73 \\
\hline 1.35 & 4.83 & 4.64 & 4.59 & 4.09 & 3.88 \\
\hline 1.40 & 4.94 & 4.74 & - & 4.16 & 4.01 \\
\hline 1.45 & 5.04 & 4.83 & - & - & 4.12 \\
\hline 1.50 & 5.12 & 4.93 & - & - & 4.22 \\
\hline 1.55 & 5.20 & 5.02 & - & - & 4.33 \\
\hline 1.60 & 5.25 & 5.11 & - & - & 4.43 \\
\hline 1.65 & 5.30 & 5.18 & - & - & 4.52 \\
\hline 1.70 & 5.33 & 5.26 & - & - & 4.61 \\
\hline 1.75 & 5.36 & 5.32 & - & - & 4.71 \\
\hline 1.80 & 5.38 & 5.39 & - & - & - \\
\hline 1.85 & - & 5.45 & - & - & - \\
\hline 1.90 & - & 5.51 & - & - & - \\
\hline 1.95 & - & 5.60 & - & - & - \\
\hline
\end{tabular}

We adopted the interval in $0.90<(V-J)_{0} \leq 1.75 \mathrm{mag}$, where at least three clusters are defined, for the application of the procedure and we evaluated $M_{J}$ absolute magnitudes for each colour. Then, we combined them with the corresponding $[\mathrm{Fe} / \mathrm{H}]$ metallicities and obtained the final calibrations. The general form of the equation for the calibrations is as follows:

$$
M_{J}=d_{0}+d_{1} X+d_{2} X^{2}
$$

where $X=[\mathrm{Fe} / \mathrm{H}]$. The absolute magnitudes estimated via Equation (10) for $85(V-J)_{o}$ colour indices and the corresponding $d_{i}(i=0,1,2)$ coefficients are given in Table 12.

\subsection{Application of the procedure}

We have two absolute magnitude calibrations, $M_{g}$ and $M_{J}$, in terms of metallicity. Hence, we can apply each of them to a set of data.

\subsubsection{Application of the $M_{g} \times[\mathrm{Fe} / \mathrm{H}]$ calibration}

The procedure is applied to four clusters with different metal abundances (M53, M3, M71, and M35). The reason of preferring stellar clusters instead of individual field dwarfs is that clusters provide absolute magnitudes for comparison with the ones estimated by the method. The choice of test clusters as well as the calibrator ones is arbitrary. Hence, we

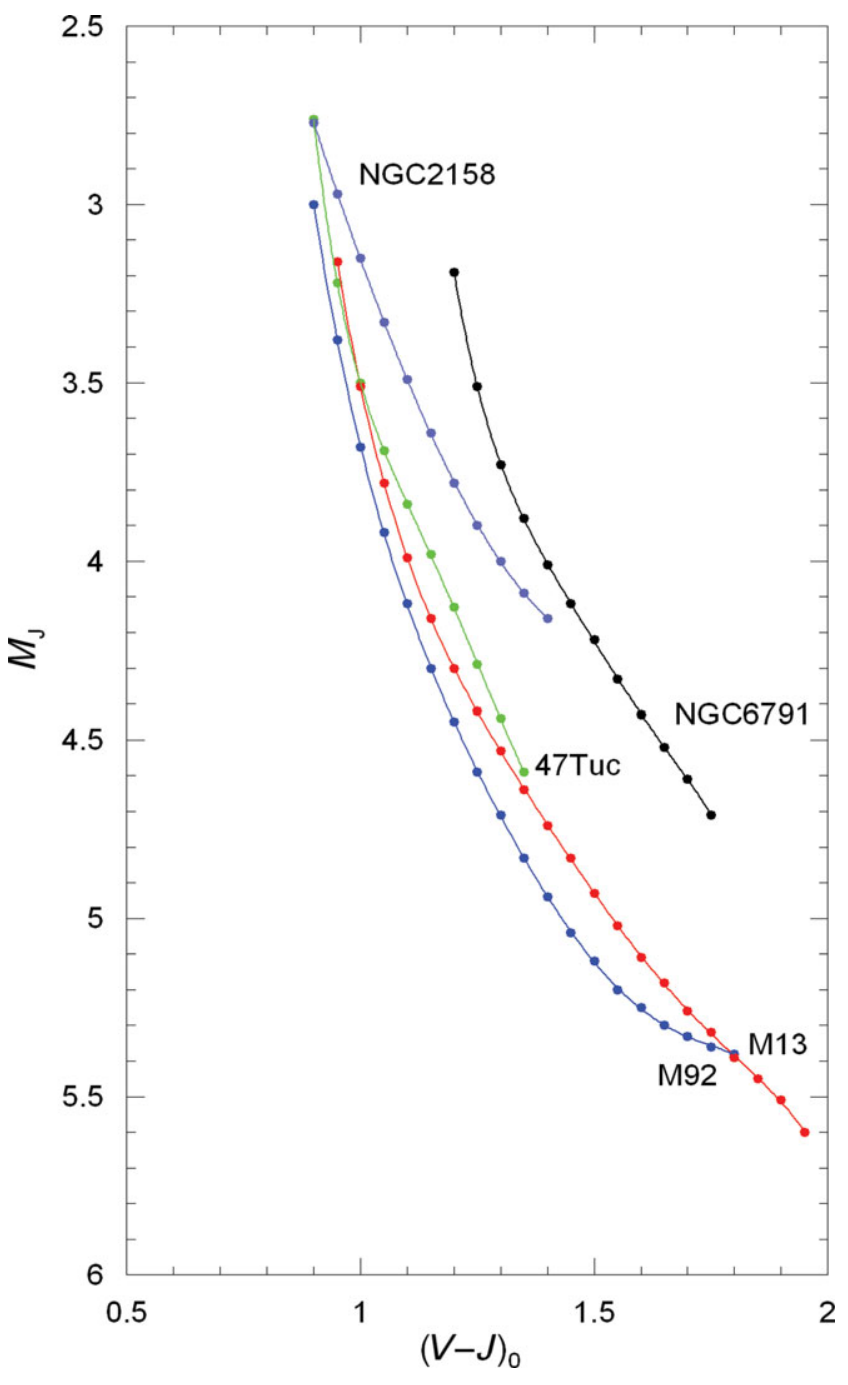

Figure 7. $M_{J} \times(V-J)_{0}$ colour-absolute magnitude diagrams for five clusters used for the absolute magnitude calibration with 2MASS.

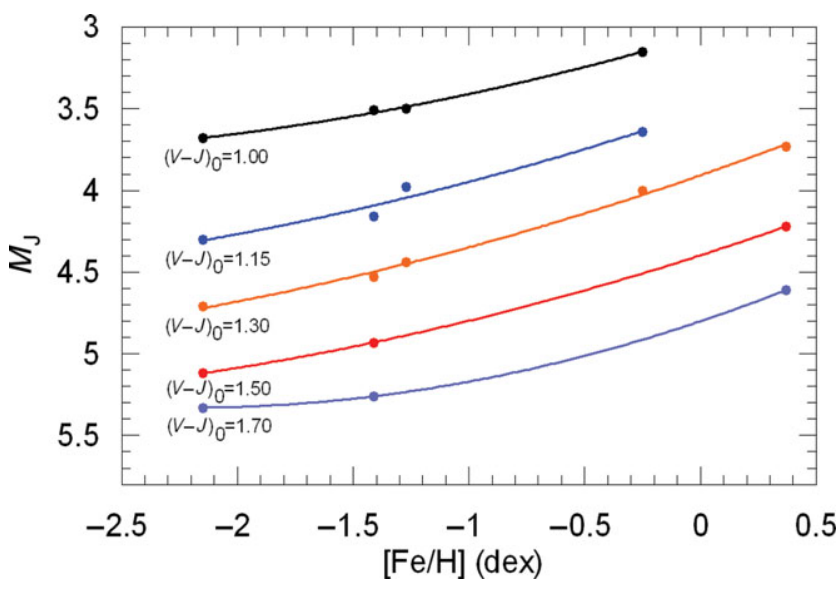

Figure 8. Calibration of the absolute magnitude $M_{J}$ as a function of metallicity $[\mathrm{Fe} / \mathrm{H}]$ for five colour indices. 
Table 11. $M_{J}$ absolute magnitudes and $[\mathrm{Fe} / \mathrm{H}]$ metallicities for five $(V-J)_{0}$ intervals.

\begin{tabular}{lcc}
\hline \hline $\begin{array}{l}(\mathrm{V}-J)_{0} \\
(\mathrm{mag})\end{array}$ & $\begin{array}{c}{[\mathrm{Fe} / \mathrm{H}]} \\
(\mathrm{dex})\end{array}$ & $\begin{array}{c}M_{J} \\
(\mathrm{mag})\end{array}$ \\
\hline 1.00 & -2.15 & 3.68 \\
& -1.41 & 3.51 \\
& -1.27 & 3.50 \\
1.15 & -0.25 & 3.15 \\
& -2.15 & 4.30 \\
& -1.41 & 4.16 \\
& -1.27 & 3.98 \\
1.30 & -0.25 & 3.64 \\
& -2.15 & 4.71 \\
& -1.41 & 4.53 \\
& -1.27 & 4.44 \\
1.50 & -0.25 & 4.00 \\
& 0.37 & 3.73 \\
& -2.15 & 5.12 \\
1.70 & -1.41 & 4.93 \\
& 0.37 & 4.22 \\
& -2.15 & 5.33 \\
& -1.41 & 5.26 \\
\hline \hline
\end{tabular}

did not involve these clusters into the calibration set but we used them to confirm the robustness of the procedure. In the case of field stars, one needs their distances or trigonometric parallaxes in addition to their $g_{0}$ and $(g-r)_{0}$ data in order to evaluate their absolute magnitudes for their comparison with the ones estimated by the procedure in our work. However, such stars are rare in the literature. The parameters of stellar clusters and $(g, g-r)$ photometric data are given in Tables 13 and 14, respectively. The colour-apparent magnitude diagrams of the Galactic clusters used for the application of the procedure are shown in Figure 9. The $g$ and the $g-r$ data of the clusters M53, M3, and M71 are taken from An et al. (2008), whereas those of M35 are transformed from the $V$ and $B-V$ data in von Hippel et al. (2002) by the reduced transformation equations of Chonis \& Gaskell (2008) given in the following:

$$
\begin{array}{r}
g=V+0.642 \times(B-V)-0.135, \\
g-r=1.094 \times(B-V)-0.248
\end{array}
$$

The reason of this choice is to obtain the $g$ and $g-r$ data for a relatively metal rich cluster with a large colour range. The metallicity of M35 is $[\mathrm{Fe} / \mathrm{H}]=-0.16 \mathrm{dex}$, and it covers the colour range of $-0.30 \leq(g-r)_{0} \leq 1.40 \mathrm{mag}$. However, the application could be carried out for the colour interval $0.35 \leq(g-r)_{0} \leq 1.25 \mathrm{mag}$, due to the restriction of our calibrations for the metallicity $[\mathrm{Fe} / \mathrm{H}]=-0.16 \mathrm{dex}$ (see Table 9).

We evaluated the $M_{g}$ absolute magnitude by Equation (8) for a set of $(g-r)_{0}$ colour indices where the clusters are defined. The results are given in Table 15. The columns refer to (1) $(g-r)_{0}$, colour index; (2) $\left(M_{g}\right)_{e v}$, the absolute magnitude estimated by the procedure; (3) $\left(M_{g}\right)_{c l}$, absolute magnitude for a cluster estimated by its colourmagnitude diagram; and (4) $\Delta M$, absolute magnitude residuals. Also, the metallicity for each cluster is indicated near the name of the cluster. The differences between the absolute magnitudes estimated by the procedure presented in this study and those evaluated via colour-magnitude diagrams of the clusters (the residuals) lie between -0.15 and +0.12 mag. The mean and the standard deviation of the residuals are $<\Delta M_{g}>=-0.002$ and $\sigma=0.065 \mathrm{mag}$, respectively. The distribution of the residuals is given in Table 16 and Figure 10.

The linear (Equation (9)) is valid for the colours $(g-$ $r)_{0} \geq 0.82$. Hence, we could apply it to only cluster M35. We used the $c_{1}$ and $c_{0}$ coefficients in Table 9 and evaluated the $\left(M_{g}\right)_{e v}$ absolute magnitudes for nine $(g-r)_{0}$ colours, i.e. $0.85,0.90,0.95,1.0,1.05,1.10,1.15,1.20$, and 1.25 mag. Then, we compared them with the corresponding ones, $\left(M_{g}\right)_{c l}$. The mean of the residuals and the standard deviation for this set of data are $\langle\Delta M>=-0.102$ and $\sigma=0.094$ mag, respectively.

\subsubsection{Application of the $M_{J} \times[\mathrm{Fe} / \mathrm{H}]$ calibration}

We applied the procedure to the cluster M5 and to a set of field stars with solar metallicity as explained in the following. The reason of choosing a cluster is that it provides absolute magnitudes for comparison with the ones estimated by the procedure. The colour excess, $E(B-V)=0.03 \mathrm{mag}$ and the metallicity $[\mathrm{Fe} / \mathrm{H}]=-1.40$ dex of the cluster are taken from Reid (1997), while for the apparent distance modulus we adopted the one of Brasseur et al. (2010), $\mu=14.45$ mag. Sarajedini et al. (2004) published the 2MASS magnitudes, $V$ magnitudes, metallicities, and parallaxes of 54 field stars. Parallaxes provide absolute magnitudes for comparison with the ones estimated by our procedure, and this is the reason of choosing this sample of stars. The range of the metallicity is $-0.45 \leq[\mathrm{Fe} / \mathrm{H}] \leq+0.35$ dex, i.e., the stars are of solar metallicity. Hence, their combination with (relatively) metalpoor stars in cluster M5 provides a good sample for the application of the procedure. Two stars, Hip 69301 and Hip 84164, are omitted due to their large relative parallax errors, $\sigma_{\pi} / \pi=0.16$ and 0.09 , respectively. Also, six stars which fall off the colour domain of our procedure, i.e. $(V-J)_{0}>1.75$ mag, could not be included into our programme. The $V-J$ colours and $J$ magnitudes of M5 cluster were de-reddened by the equations given in Section 3.1.2, while all the magnitudes in Sarajedini et al. (2004) were assumed as unaffected from the interstellar extinction due to the proximity of the field stars to the Sun, $d<75 \mathrm{pc}$. The results are given in Table 17 and Figure 11. The data of the field stars in columns (1)-(6) are original, while those in (7)-(9) are evaluated in this paper. The distance (d) was evaluated by using the corresponding parallax, and the absolute magnitude $M_{J}$ was calculated by the well known Pogson formula. 
Table 12. $M_{J}$ absolute magnitudes estimated for five Galactic clusters and the numerical values of $d_{i}(i=0,1,2)$ coefficients in Equation (11)

\begin{tabular}{|c|c|c|c|c|c|c|c|c|c|c|}
\hline $\begin{array}{l}\text { Cluster } \\
(V-J)_{0}\end{array}$ & $\begin{array}{l}\text { M92 } \\
M_{J}\end{array}$ & $\begin{array}{c}\mathrm{M} 13 \\
M_{J}\end{array}$ & $\begin{array}{c}47 \text { Tuc } \\
M_{J}\end{array}$ & $\begin{array}{c}\text { NGC } 2158 \\
M_{J}\end{array}$ & $\begin{array}{c}\text { NGC } 6791 \\
M_{J}\end{array}$ & $d_{2}$ & $d_{1}$ & $d_{0}$ & $R^{2}$ & $\begin{array}{c}{[\mathrm{Fe} / \mathrm{H}]} \\
\text { interval (dex) }\end{array}$ \\
\hline 0.91 & 3.08 & - & 2.87 & 2.81 & - & 0.0946 & 0.0856 & 2.8282 & 1.000 & {$[-2.15,-0.25]$} \\
\hline 0.92 & 3.16 & - & 2.97 & 2.85 & - & 0.0507 & -0.0411 & 2.8382 & 1.000 & {$[-2.15,-0.25]$} \\
\hline 0.93 & 3.24 & - & 3.06 & 2.89 & - & 0.0150 & -0.1461 & 2.8529 & 1.000 & {$[-2.15,-0.25]$} \\
\hline 0.94 & 3.31 & - & 3.14 & 2.93 & - & -0.0133 & -0.2318 & 2.8715 & 1.000 & {$[-2.15,-0.25]$} \\
\hline 0.95 & 3.38 & 3.16 & 3.22 & 2.97 & - & 0.0122 & -0.1831 & 2.9226 & 0.953 & {$[-2.15,-0.25]$} \\
\hline 0.96 & 3.44 & 3.24 & 3.29 & 3.00 & - & -0.0086 & -0.2482 & 2.9450 & 0.967 & {$[-2.15,-0.25]$} \\
\hline 0.97 & 3.51 & 3.31 & 3.35 & 3.04 & - & -0.0256 & -0.3029 & 2.9694 & 0.978 & {$[-2.15,-0.25]$} \\
\hline 0.98 & 3.57 & 3.38 & 3.40 & 3.08 & - & -0.0394 & -0.3485 & 2.9954 & 0.987 & {$[-2.15,-0.25]$} \\
\hline 0.99 & 3.62 & 3.45 & 3.45 & 3.12 & - & -0.0502 & -0.3860 & 3.0229 & 0.993 & {$[-2.15,-0.25]$} \\
\hline 1.00 & 3.68 & 3.51 & 3.50 & 3.15 & - & -0.0585 & -0.4164 & 3.0516 & 0.997 & {$[-2.15,-0.25]$} \\
\hline 1.01 & 3.73 & 3.57 & 3.54 & 3.19 & - & -0.0646 & -0.4405 & 3.0813 & 0.999 & {$[-2.15,-0.25]$} \\
\hline 1.02 & 3.78 & 3.62 & 3.58 & 3.22 & - & -0.0688 & -0.4593 & 3.1119 & 1.000 & {$[-2.15,-0.25]$} \\
\hline 1.03 & 3.83 & 3.68 & 3.62 & 3.26 & - & -0.0714 & -0.4734 & 3.1431 & 0.999 & {$[-2.15,-0.25]$} \\
\hline 1.04 & 3.88 & 3.73 & 3.66 & 3.29 & - & -0.0728 & -0.4836 & 3.1747 & 0.997 & {$[-2.15,-0.25]$} \\
\hline 1.05 & 3.92 & 3.78 & 3.69 & 3.33 & - & -0.0731 & -0.4906 & 3.2067 & 0.994 & {$[-2.15,-0.25]$} \\
\hline 1.06 & 3.96 & 3.82 & 3.72 & 3.36 & - & -0.0725 & -0.4949 & 3.2389 & 0.991 & {$[-2.15,-0.25]$} \\
\hline 1.07 & 4.01 & 3.87 & 3.75 & 3.39 & - & -0.0714 & -0.4970 & 3.2711 & 0.987 & {$[-2.15,-0.25]$} \\
\hline 1.08 & 4.05 & 3.91 & 3.78 & 3.43 & - & -0.0697 & -0.4975 & 3.3033 & 0.983 & {$[-2.15,-0.25]$} \\
\hline 1.09 & 4.09 & 3.95 & 3.81 & 3.46 & - & -0.0678 & -0.4968 & 3.3353 & 0.980 & {$[-2.15,-0.25]$} \\
\hline 1.10 & 4.12 & 3.99 & 3.84 & 3.49 & - & -0.0657 & -0.4953 & 3.3670 & 0.976 & {$[-2.15,-0.25]$} \\
\hline 1.11 & 4.16 & 4.02 & 3.87 & 3.52 & - & -0.0635 & -0.4933 & 3.3984 & 0.973 & {$[-2.15,-0.25]$} \\
\hline 1.12 & 4.20 & 4.06 & 3.90 & 3.55 & - & -0.0614 & -0.4911 & 3.4294 & 0.971 & {$[-2.15,-0.25]$} \\
\hline 1.13 & 4.23 & 4.09 & 3.93 & 3.58 & - & -0.0595 & -0.4889 & 3.4599 & 0.969 & {$[-2.15,-0.25]$} \\
\hline 1.14 & 4.26 & 4.13 & 3.95 & 3.61 & - & -0.0578 & -0.4871 & 3.4898 & 0.967 & {$[-2.15,-0.25]$} \\
\hline 1.15 & 4.30 & 4.16 & 3.98 & 3.64 & - & -0.0564 & -0.4857 & 3.5192 & 0.967 & {$[-2.15,-0.25]$} \\
\hline 1.16 & 4.33 & 4.19 & 4.01 & 3.67 & - & -0.0554 & -0.4850 & 3.5478 & 0.967 & {$[-2.15,-0.25]$} \\
\hline 1.17 & 4.36 & 4.22 & 4.04 & 3.70 & - & -0.0547 & -0.4850 & 3.5758 & 0.967 & {$[-2.15,-0.25]$} \\
\hline 1.18 & 4.39 & 4.24 & 4.07 & 3.72 & - & -0.0544 & -0.4859 & 3.6030 & 0.969 & {$[-2.15,-0.25]$} \\
\hline 1.19 & 4.42 & 4.27 & 4.10 & 3.75 & - & -0.0546 & -0.4878 & 3.6295 & 0.970 & {$[-2.15,-0.25]$} \\
\hline 1.20 & 4.45 & 4.30 & 4.13 & 3.78 & 3.19 & -0.1490 & -0.7434 & 3.5189 & 0.983 & {$[-2.15,0.37]$} \\
\hline 1.21 & 4.48 & 4.32 & 4.16 & 3.80 & 3.26 & -0.1324 & -0.6998 & 3.5693 & 0.986 & {$[-2.15,0.37]$} \\
\hline 1.22 & 4.51 & 4.35 & 4.19 & 3.83 & 3.33 & -0.1179 & -0.6617 & 3.6165 & 0.989 & {$[-2.15,0.37]$} \\
\hline 1.23 & 4.53 & 4.37 & 4.22 & 3.85 & 3.40 & -0.1052 & -0.6284 & 3.6609 & 0.991 & {$[-2.15,0.37]$} \\
\hline 1.24 & 4.56 & 4.40 & 4.26 & 3.88 & 3.45 & -0.0942 & -0.5996 & 3.7025 & 0.993 & {$[-2.15,0.37]$} \\
\hline 1.25 & 4.59 & 4.42 & 4.29 & 3.90 & 3.51 & -0.0847 & -0.5748 & 3.7416 & 0.995 & {$[-2.15,0.37]$} \\
\hline 1.26 & 4.61 & 4.44 & 4.32 & 3.92 & 3.56 & -0.0765 & -0.5536 & 3.7785 & 0.996 & {$[-2.15,0.37]$} \\
\hline 1.27 & 4.64 & 4.46 & 4.35 & 3.94 & 3.61 & -0.0696 & -0.5356 & 3.8133 & 0.997 & {$[-2.15,0.37]$} \\
\hline 1.28 & 4.67 & 4.49 & 4.38 & 3.96 & 3.65 & -0.0636 & -0.5205 & 3.8462 & 0.997 & {$[-2.15,0.37]$} \\
\hline 1.29 & 4.69 & 4.51 & 4.41 & 3.98 & 3.69 & -0.0586 & -0.5079 & 3.8773 & 0.998 & {$[-2.15,0.37]$} \\
\hline 1.30 & 4.71 & 4.53 & 4.44 & 4.00 & 3.73 & -0.0544 & -0.4975 & 3.9069 & 0.997 & {$[-2.15,0.37]$} \\
\hline 1.31 & 4.74 & 4.55 & 4.47 & 4.02 & 3.76 & -0.0509 & -0.4890 & 3.9349 & 0.997 & {$[-2.15,0.37]$} \\
\hline 1.32 & 4.76 & 4.57 & 4.50 & - & 3.80 & -0.0614 & -0.4941 & 3.9860 & 0.999 & {$[-2.15,0.37]$} \\
\hline 1.33 & 4.79 & 4.59 & 4.53 & - & 3.83 & -0.0614 & -0.4908 & 4.0159 & 1.000 & {$[-2.15,0.37]$} \\
\hline 1.34 & 4.81 & 4.61 & 4.56 & - & 3.86 & -0.0618 & -0.4888 & 4.0447 & 1.000 & {$[-2.15,0.37]$} \\
\hline 1.35 & 4.83 & 4.64 & 4.59 & - & 3.88 & -0.0625 & -0.4877 & 4.0725 & 1.000 & {$[-2.15,0.37]$} \\
\hline 1.36 & 4.85 & 4.66 & - & - & 3.91 & -0.0635 & -0.4876 & 4.0995 & 1.000 & {$[-2.15,0.37]$} \\
\hline 1.37 & 4.88 & 4.68 & - & - & 3.94 & -0.0647 & -0.4882 & 4.1256 & 1.000 & {$[-2.15,0.37]$} \\
\hline 1.38 & 4.90 & 4.70 & - & - & 3.96 & -0.0556 & -0.4712 & 4.1426 & 1.000 & {$[-2.15,0.37]$} \\
\hline 1.39 & 4.92 & 4.72 & - & - & 3.98 & -0.0541 & -0.4674 & 4.1650 & 1.000 & {$[-2.15,0.37]$} \\
\hline 1.40 & 4.94 & 4.74 & - & - & 4.01 & -0.0528 & -0.4642 & 4.1870 & 1.000 & {$[-2.15,0.37]$} \\
\hline 1.41 & 4.96 & 4.76 & - & - & 4.03 & -0.0518 & -0.4614 & 4.2087 & 1.000 & {$[-2.15,0.37]$} \\
\hline 1.42 & 4.98 & 4.78 & - & - & 4.05 & -0.0511 & -0.4591 & 4.2301 & 1.000 & {$[-2.15,0.37]$} \\
\hline 1.43 & 5.00 & 4.80 & - & - & 4.08 & -0.0506 & -0.4572 & 4.2514 & 1.000 & {$[-2.15,0.37]$} \\
\hline 1.44 & 5.02 & 4.81 & - & - & 4.10 & -0.0503 & -0.4556 & 4.2725 & 1.000 & {$[-2.15,0.37]$} \\
\hline 1.45 & 5.04 & 4.83 & - & - & 4.12 & -0.0502 & -0.4543 & 4.2935 & 1.000 & {$[-2.15,0.37]$} \\
\hline 1.46 & 5.06 & 4.85 & - & - & 4.14 & -0.0503 & -0.4533 & 4.3145 & 1.000 & {$[-2.15,0.37]$} \\
\hline 1.47 & 5.07 & 4.87 & - & - & 4.16 & -0.0507 & -0.4525 & 4.3354 & 1.000 & {$[-2.15,0.37]$} \\
\hline 1.48 & 5.09 & 4.89 & - & - & 4.18 & -0.0512 & -0.4519 & 4.3563 & 1.000 & {$[-2.15,0.37]$} \\
\hline 1.49 & 5.11 & 4.91 & - & - & 4.20 & -0.0519 & -0.4514 & 4.3771 & 1.000 & {$[-2.15,0.37]$} \\
\hline 1.50 & 5.12 & 4.93 & - & - & 4.22 & -0.0528 & -0.4511 & 4.3980 & 1.000 & {$[-2.15,0.37]$} \\
\hline
\end{tabular}


Table 12. Continued.

\begin{tabular}{|c|c|c|c|c|c|c|c|c|c|c|}
\hline $\begin{array}{l}\text { Cluster } \\
(V-J)_{0}\end{array}$ & $\begin{array}{c}\text { M92 } \\
M_{J}\end{array}$ & $\begin{array}{c}\text { M13 } \\
M_{J}\end{array}$ & $\begin{array}{c}47 \text { Tuc } \\
M_{J}\end{array}$ & $\begin{array}{c}\text { NGC } 2158 \\
M_{J}\end{array}$ & $\begin{array}{c}\text { NGC } 6791 \\
M_{J}\end{array}$ & $d_{2}$ & $d_{1}$ & $d_{0}$ & $R^{2}$ & $\begin{array}{c}{[\mathrm{Fe} / \mathrm{H}]} \\
\text { interval (dex) }\end{array}$ \\
\hline 1.51 & 5.14 & 4.95 & - & - & 4.24 & -0.0539 & -0.4510 & 4.4188 & 1.000 & {$[-2.15,0.37]$} \\
\hline 1.52 & 5.15 & 4.97 & - & - & 4.27 & -0.0551 & -0.4510 & 4.4397 & 1.000 & {$[-2.15,0.37]$} \\
\hline 1.53 & 5.17 & 4.98 & - & - & 4.29 & -0.0566 & -0.4512 & 4.4605 & 1.000 & {$[-2.15,0.37]$} \\
\hline 1.54 & 5.18 & 5.00 & - & - & 4.31 & -0.0582 & -0.4515 & 4.4813 & 1.000 & {$[-2.15,0.37]$} \\
\hline 1.55 & 5.20 & 5.02 & - & - & 4.33 & -0.0600 & -0.4519 & 4.5020 & 1.000 & {$[-2.15,0.37]$} \\
\hline 1.56 & 5.21 & 5.04 & - & - & 4.35 & -0.0620 & -0.4525 & 4.5227 & 1.000 & {$[-2.15,0.37]$} \\
\hline 1.57 & 5.22 & 5.05 & - & - & 4.37 & -0.0641 & -0.4532 & 4.5433 & 1.000 & {$[-2.15,0.37]$} \\
\hline 1.58 & 5.23 & 5.07 & - & - & 4.39 & -0.0665 & -0.4541 & 4.5638 & 1.000 & {$[-2.15,0.37]$} \\
\hline 1.59 & 5.24 & 5.09 & - & - & 4.41 & -0.0689 & -0.4550 & 4.5843 & 1.000 & {$[-2.15,0.37]$} \\
\hline 1.60 & 5.25 & 5.11 & - & - & 4.43 & -0.0715 & -0.4561 & 4.6046 & 1.000 & {$[-2.15,0.37]$} \\
\hline 1.61 & 5.26 & 5.12 & - & - & 4.45 & -0.0743 & -0.4573 & 4.6248 & 1.000 & {$[-2.15,0.37]$} \\
\hline 1.62 & 5.27 & 5.14 & - & - & 4.46 & -0.0772 & -0.4587 & 4.6448 & 1.000 & {$[-2.15,0.37]$} \\
\hline 1.63 & 5.28 & 5.15 & - & - & 4.48 & -0.0803 & -0.4601 & 4.6647 & 1.000 & {$[-2.15,0.37]$} \\
\hline 1.64 & 5.29 & 5.17 & - & - & 4.50 & -0.0834 & -0.4616 & 4.6845 & 1.000 & {$[-2.15,0.37]$} \\
\hline 1.65 & 5.30 & 5.18 & - & - & 4.52 & -0.0867 & -0.4631 & 4.7041 & 1.000 & {$[-2.15,0.37]$} \\
\hline 1.66 & 5.31 & 5.20 & - & - & 4.54 & -0.0900 & -0.4647 & 4.7235 & 1.000 & {$[-2.15,0.37]$} \\
\hline 1.67 & 5.31 & 5.21 & - & - & 4.56 & -0.0934 & -0.4663 & 4.7429 & 1.000 & {$[-2.15,0.37]$} \\
\hline 1.68 & 5.32 & 5.23 & - & - & 4.58 & -0.0968 & -0.4678 & 4.7621 & 1.000 & {$[-2.15,0.37]$} \\
\hline 1.69 & 5.33 & 5.24 & - & - & 4.59 & -0.1003 & -0.4692 & 4.7812 & 1.000 & {$[-2.15,0.37]$} \\
\hline 1.70 & 5.33 & 5.26 & - & - & 4.61 & -0.1038 & -0.4705 & 4.8002 & 1.000 & {$[-2.15,0.37]$} \\
\hline 1.71 & 5.34 & 5.27 & - & - & 4.63 & -0.1072 & -0.4715 & 4.8193 & 1.000 & {$[-2.15,0.37]$} \\
\hline 1.72 & 5.34 & 5.28 & - & - & 4.65 & -0.1105 & -0.4723 & 4.8384 & 1.000 & {$[-2.15,0.37]$} \\
\hline 1.73 & 5.35 & 5.30 & - & - & 4.67 & -0.1137 & -0.4726 & 4.8575 & 1.000 & {$[-2.15,0.37]$} \\
\hline 1.74 & 5.35 & 5.31 & - & - & 4.69 & -0.1168 & -0.4725 & 4.8768 & 1.000 & {$[-2.15,0.37]$} \\
\hline 1.75 & 5.36 & 5.32 & - & - & 4.71 & -0.1197 & -0.4718 & 4.8963 & 1.000 & {$[-2.15,0.37]$} \\
\hline
\end{tabular}

Table 13. Data for the clusters used for the application of the procedure with SDSS.

\begin{tabular}{lcccc}
\hline \hline Cluster & $\begin{array}{c}E(B-V) \\
(\mathrm{mag})\end{array}$ & $\begin{array}{c}\mu_{0} \\
(\mathrm{mag})\end{array}$ & $\begin{array}{c}{[\mathrm{Fe} / \mathrm{H}]} \\
(\mathrm{dex})\end{array}$ & Ref \\
\hline M53 & 0.021 & 16.21 & -2.06 & 1,2 \\
M3 & 0.01 & 14.83 & -1.46 & $3,4,5$ \\
M71 & 0.32 & 13.02 & -0.73 & 3 \\
M35 & 0.19 & 9.62 & -0.16 & 6 \\
\hline \hline
\end{tabular}

(1) Smolinski et al. (2011), (2) Ferro et al. (2011), (3) An et al. (2008), (4) Saad \& Lee (2001), (5) Di Criscienzo, Marconi, \& Caputo (2004), (6) Sarajedini, Mathieu, \& Platais (2003)

We evaluated the $M_{J}$ absolute magnitude by Equation (11) for two sets of $(V-J)_{0}$ colour indices. The first set covers the domain of the cluster M5 (Table 18a), while the second one consists of $(V-J)_{0}$ colour indices of the field stars (Table $18 \mathrm{~b})$. The columns in Table 18a refer to (1) $(V-J)_{0}$ colour index, (2) $\left(M_{J}\right)_{c l}$, the absolute magnitude estimated by the combination of the colour-magnitude diagram and the true distance modulus of the cluster M5, (3) $\left(M_{J}\right)_{e v}$, the absolute magnitude estimated by the procedure, and (4) $\Delta \mathrm{M}$, absolute magnitude residuals. For metallicity, we adopted the one of cluster M5, i.e. $[\mathrm{Fe} / \mathrm{H}]=-1.40$ dex. The columns in Table $18 \mathrm{~b}$ refer to (1) $(V-J)_{0}$ colour index of the field star, (2) $\left(M_{J}\right)_{\pi}$, the absolute magnitude estimated by the parallax of the field star, (3) $[\mathrm{Fe} / \mathrm{H}]$, the metallicity of the field star, (4) $\left(M_{J}\right)_{e v}$, the absolute magnitude estimated by the procedure, and (5) $\Delta M$, absolute magnitude residuals. The total residuals lie between -0.29 and +0.39 mag. The extreme residuals $\Delta M=-1.03$ and +0.56 mag which are marked in boldface in Table 18b correspond to stars Hip 84164 and Hip 69301 whose relative parallax errors are high, as mentioned above. Hence, they are not considered in the calculations of the mean residual and standard deviation. However, the range of $87 \%$ of them is rather shorter, i.e. $-0.20<\Delta M \leq+0.20 \mathrm{mag}$. The mean and the standard deviation of all residuals, except the two extreme ones, are $\left\langle\Delta M_{J}\right\rangle=0.05$ and $\sigma=0.13$ mag. The distribution of the residuals is also given in Table 19 and Figure 12. We state that the cluster fiducials provide more accurate absolute magnitudes than the ones for the field stars. Actually, the mean and the standard deviation of the residuals for the fiducials are $\left\langle\Delta M_{J}\right\rangle=0.04$ and $\sigma=0.05$ mag, whereas those for the field stars are $\left\langle\Delta M_{J}\right\rangle=0.06$, $\sigma=0.16 \mathrm{mag}$, respectively.

\section{SUMMARY AND DISCUSSION}

We presented two absolute magnitude calibrations for dwarfs based on the colour magnitude diagrams of Galactic clusters with different metallicities. For the calibration of $M_{g}$, we used the clusters M92, M13, M5, NGC 2420, M67, and NGC 6791. We combined the calibrations between $g_{0}$ and $(g-r)_{0}$ for each cluster with their true distance modulus and evaluated a set of absolute magnitudes for the $(g-r)_{0}$ range of each cluster. Then, we fitted the $M_{g}$ absolute magnitudes in terms 
Table 14. Fiducial dwarf sequence for the galactic clusters used in the application of the procedure with SDSS.

\begin{tabular}{|c|c|c|c|c|c|c|c|c|c|}
\hline$g-r$ & $g$ & $(g-r)_{0}$ & $g_{0}$ & $B-V$ & V & $(B-V)_{0}$ & $V_{0}$ & $(g-r)_{0}$ & $g_{0}$ \\
\hline \multicolumn{4}{|c|}{ M53 } & \multicolumn{6}{|c|}{ M35 } \\
\hline 0.263 & 21.113 & 0.241 & 21.035 & 0.70 & 14.42 & 0.51 & 13.83 & 0.31 & 14.02 \\
\hline 0.291 & 21.441 & 0.269 & 21.363 & 0.80 & 15.06 & 0.61 & 14.47 & 0.42 & 14.73 \\
\hline 0.324 & 21.774 & 0.302 & 21.696 & 0.90 & 15.61 & 0.71 & 15.02 & 0.53 & 15.34 \\
\hline 0.362 & 22.112 & 0.340 & 22.034 & 1.00 & 16.00 & 0.81 & 15.41 & 0.64 & 15.80 \\
\hline \multicolumn{4}{|c|}{ M71 } & 1.10 & 16.52 & 0.91 & 15.93 & 0.75 & 16.38 \\
\hline 0.658 & 19.008 & 0.320 & 17.819 & 1.19 & 16.85 & 1.00 & 16.26 & 0.85 & 16.77 \\
\hline 0.683 & 19.333 & 0.345 & 18.144 & 1.31 & 17.30 & 1.12 & 16.71 & 0.98 & 17.30 \\
\hline 0.716 & 19.666 & 0.378 & 18.477 & 1.35 & 17.50 & 1.16 & 16.91 & 1.02 & 17.52 \\
\hline 0.757 & 20.007 & 0.419 & 18.818 & 1.55 & 18.40 & 1.36 & 17.81 & 1.24 & 18.55 \\
\hline \multicolumn{4}{|c|}{ M3 } & 1.71 & 19.10 & 1.52 & 18.51 & 1.41 & 19.35 \\
\hline 0.357 & 20.507 & 0.3464 & 20.470 & - & - & - & - & - & - \\
\hline 0.400 & 20.850 & 0.3894 & 20.813 & - & - & - & - & - & - \\
\hline 0.451 & 21.201 & 0.4404 & 21.164 & - & - & - & - & - & - \\
\hline 0.509 & 21.559 & 0.4984 & 21.522 & - & - & - & - & - & - \\
\hline 0.574 & 21.924 & 0.5634 & 21.887 & - & - & - & - & - & - \\
\hline 0.640 & 22.290 & 0.6294 & 22.253 & - & - & - & - & - & - \\
\hline
\end{tabular}

Table 15. Absolute magnitudes $\left(\left(M_{g}\right)_{e v}\right)$ and residuals $(\Delta M)$ estimated by the procedure explained in our work. $\left(M_{g}\right)_{c l}$ denotes the absolute magnitude evaluated by means of colour - magnitude diagram of the cluster.

\begin{tabular}{|c|c|c|c|c|c|c|c|c|c|c|c|}
\hline $\begin{array}{l}1 \\
(g-r)_{0}\end{array}$ & $\begin{array}{c}2 \\
\left(M_{g}\right)_{e v}\end{array}$ & $\begin{array}{c}3 \\
\left(M_{g}\right)_{c l}\end{array}$ & $\begin{array}{c}4 \\
\Delta M\end{array}$ & $\begin{array}{c}1 \\
(g-r)_{0}\end{array}$ & $\begin{array}{c}2 \\
\left(M_{g}\right)_{e v}\end{array}$ & $\begin{array}{c}3 \\
\left(M_{g}\right)_{c l}\end{array}$ & $\begin{array}{c}4 \\
\Delta M\end{array}$ & $\begin{array}{c}1 \\
(g-r)_{0}\end{array}$ & $\begin{array}{c}2 \\
\left(M_{g}\right)_{e v}\end{array}$ & $\begin{array}{c}3 \\
\left(M_{g}\right)_{c l}\end{array}$ & $\begin{array}{c}4 \\
\Delta M\end{array}$ \\
\hline \multicolumn{4}{|c|}{$\operatorname{M} 53([\mathrm{Fe} / \mathrm{H}]=-2.06 \mathrm{dex})$} & \multicolumn{4}{|c|}{$\mathrm{M} 3([\mathrm{Fe} / \mathrm{H}]=-1.46 \mathrm{dex})$} & \multicolumn{4}{|c|}{$\operatorname{M71}([\mathrm{Fe} / \mathrm{H}]=-0.73 \mathrm{dex})$} \\
\hline 0.25 & 4.932 & 4.936 & -0.004 & 0.35 & 5.718 & 5.671 & 0.047 & 0.32 & 4.920 & 4.806 & 0.114 \\
\hline 0.26 & 5.051 & 5.051 & -0.001 & 0.36 & 5.792 & 5.753 & 0.039 & 0.33 & 5.008 & 4.936 & 0.072 \\
\hline 0.27 & 5.161 & 5.163 & -0.002 & 0.37 & 5.863 & 5.833 & 0.031 & 0.34 & 5.092 & 5.059 & 0.033 \\
\hline 0.28 & 5.265 & 5.270 & -0.005 & 0.38 & 5.931 & 5.910 & 0.021 & 0.35 & 5.199 & 5.176 & 0.023 \\
\hline 0.29 & 5.363 & 5.373 & -0.010 & 0.39 & 5.997 & 5.986 & 0.011 & 0.36 & 5.279 & 5.286 & -0.007 \\
\hline 0.30 & 5.457 & 5.471 & -0.014 & 0.40 & 6.060 & 6.059 & 0.001 & 0.37 & 5.356 & 5.389 & -0.033 \\
\hline 0.31 & 5.548 & 5.566 & -0.018 & 0.41 & 6.121 & 6.130 & -0.009 & 0.38 & 5.430 & 5.485 & -0.055 \\
\hline 0.32 & 5.634 & 5.656 & -0.022 & 0.42 & 6.181 & 6.199 & -0.018 & 0.39 & 5.503 & 5.575 & -0.072 \\
\hline 0.33 & 5.718 & 5.743 & -0.025 & 0.43 & 6.241 & 6.267 & -0.026 & 0.40 & 5.574 & 5.658 & -0.085 \\
\hline 0.34 & 5.797 & 5.825 & -0.027 & 0.44 & 6.299 & 6.333 & -0.034 & 0.41 & 5.643 & 5.735 & -0.092 \\
\hline- & - & - & - & 0.45 & 6.359 & 6.397 & -0.039 & - & - & - & - \\
\hline- & - & - & - & 0.46 & 6.419 & 6.460 & -0.042 & - & - & - & - \\
\hline \multicolumn{4}{|c|}{$\mathrm{M} 35([\mathrm{Fe} / \mathrm{H}]=-0.16 \mathrm{dex})$} & \multicolumn{4}{|c|}{ M35 (cont.) } & \multicolumn{4}{|c|}{ M35 (cont.) } \\
\hline 0.35 & 4.55 & 4.68 & -0.127 & 0.70 & 6.59 & 6.51 & 0.082 & 1.00 & 7.82 & 7.81 & 0.009 \\
\hline 0.40 & 4.96 & 4.98 & -0.019 & 0.75 & 6.81 & 6.74 & 0.069 & 1.05 & 7.99 & 8.03 & -0.038 \\
\hline 0.45 & 5.31 & 5.26 & 0.053 & 0.80 & 7.03 & 6.96 & 0.067 & 1.10 & 8.16 & 8.24 & -0.085 \\
\hline 0.50 & 5.62 & 5.53 & 0.086 & 0.85 & 7.29 & 7.17 & 0.117 & 1.15 & 8.34 & 8.46 & -0.126 \\
\hline 0.55 & 5.85 & 5.79 & 0.056 & 0.90 & 7.48 & 7.39 & 0.089 & 1.20 & 8.54 & 8.69 & -0.154 \\
\hline 0.60 & 6.12 & 6.04 & 0.077 & 0.95 & 7.65 & 7.60 & 0.053 & 1.25 & 8.77 & 8.92 & -0.155 \\
\hline 0.65 & 6.37 & 6.28 & 0.085 & - & - & - & - & - & - & - & - \\
\hline
\end{tabular}

of the iron metallicity, $[\mathrm{Fe} / \mathrm{H}]$, by a quadratic polynomial for a given $(g-r)_{0}$ colour index. Our absolute magnitude calibrations cover the range $0.25 \leq(g-r)_{0} \leq 1.25 \mathrm{mag}$.

We applied the procedure to another set of Galactic cluster, i.e. M53, M3, M71, and M35 and compared the absolute magnitudes estimated by this procedure with those evaluated via a combination of the fiducial $g_{0},(g-r)_{0}$ sequence and the true distance modulus for each cluster. The residuals lie between -0.15 and +0.12 mag, and their mean and standard deviations are $\langle\Delta M\rangle=-0.002$ and $\sigma=0.065$ mag. The range of the residuals estimated for the red giants with SDSS was $-0.28 \leq \Delta M \leq+0.43$ mag (Karaali et al. 2013a), larger than the one for the dwarfs estimated in this study. Also, their mean and standard deviation were larger, i.e. $\langle\Delta M>=0.169$ and $\sigma=0.140$. The difference between two sets of residuals originates mainly from the different trends of the colour-magnitude diagrams of red giants and dwarfs, i.e., the colour-magnitude diagram of red 


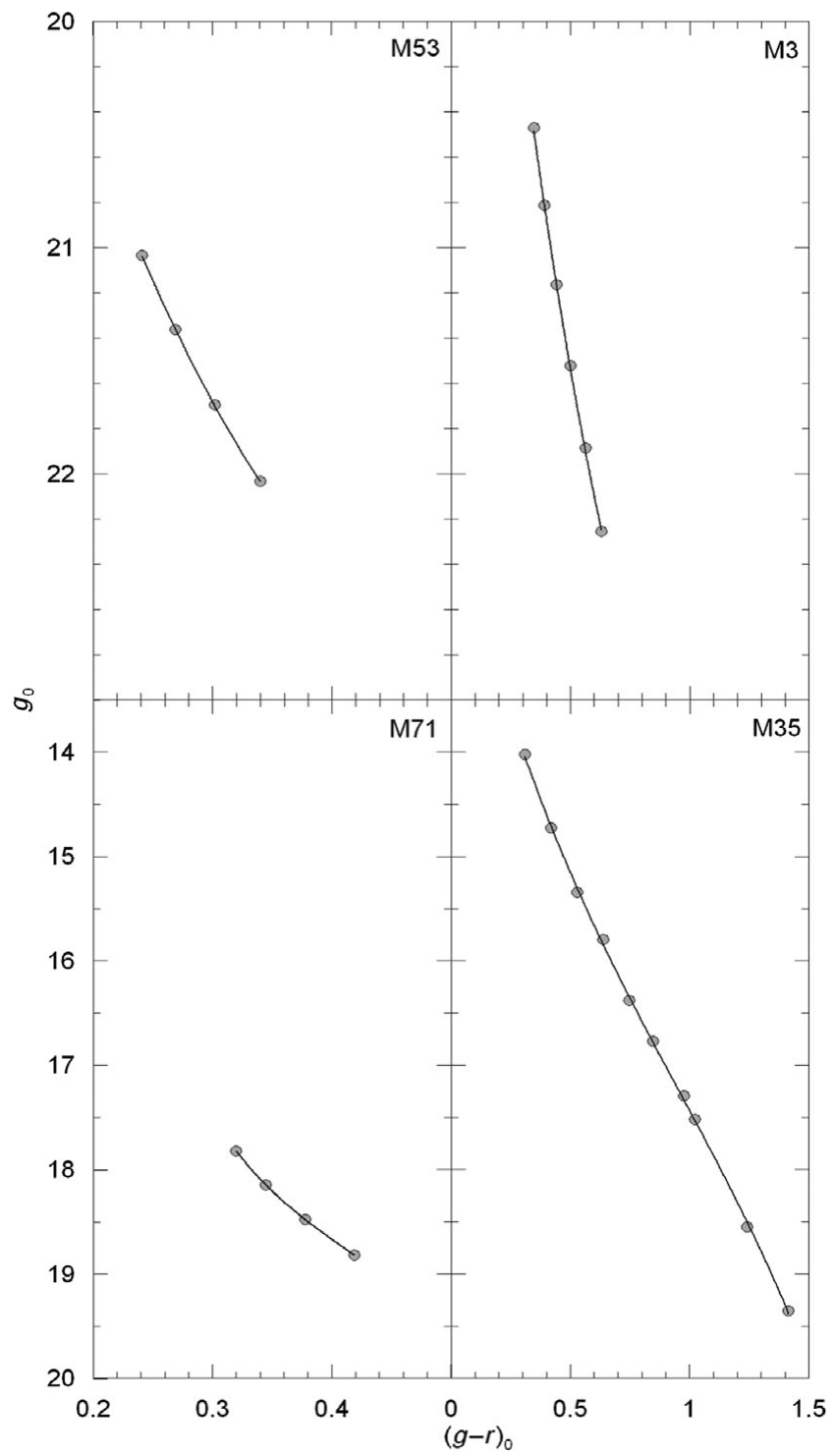

Figure 9. $g_{0} \times(g-r)_{0}$ colour-apparent magnitude diagrams for the Galactic clusters used for the application of the procedure.

Table 16. Distribution of the residuals. $N$ denotes the number of stars.

\begin{tabular}{lrr}
\hline \hline$\Delta M_{g}$-interval & $\left\langle\Delta M_{g}\right\rangle$ & $N$ \\
\hline$[-0.15,-0.10]$ & -0.140 & 4 \\
{$[-0.10,-0.05]$} & -0.078 & 5 \\
{$[-0.05,0.00]$} & -0.020 & 20 \\
{$[0.00,0.05]$} & 0.024 & 9 \\
{$[0.05,0.10]$} & 0.072 & 11 \\
{$[0.10,0.15]$} & 0.115 & 2 \\
\hline \hline
\end{tabular}

giants for a given cluster is steeper than the one for dwarfs of the same cluster, and any error in $(g-r)_{0}$ results in larger colour errors within the steeper diagram.

The range of the $(B-V)_{0}$ colour in Karaali et al. (2003) who estimated absolute magnitudes for dwarfs by absolute magnitude offsets is $0.3 \leq(B-V)_{0} \leq 1.1$ mag which corresponds to $0.1 \leq(g-r)_{0} \leq 0.9 \mathrm{mag}$, shorter than the one cited in our study, i.e. $0.25 \leq(g-r)_{0} \leq 1.25 \mathrm{mag}$. That is, there is an improvement on our procedure with respect to the one of Karaali et al. (2003).

For the calibration of $M_{J}$, we used the clusters M92, M13, 47 Tuc, NGC 2158 and NGC 6791. We combined the calibrations between $J_{0}$ and $(V-J)_{0}$ for each cluster with their true distance modulus and evaluated a set of absolute magnitudes for the $(V-J)_{0}$ range of each cluster. Then, we calibrated the $M_{J}$ absolute magnitudes in terms of the iron metallicity, $[\mathrm{Fe} / \mathrm{H}]$, by a quadratic polynomial for all cluster. Our absolute magnitude calibrations cover the range $0.90<(V-J)_{0} \leq 1.75 \mathrm{mag}$.

This is our fourth paper devoted to the calibration of the absolute magnitudes based on the colour-magnitude diagrams of Galactic clusters, and it is the first time that we 
Table 17. Data for the cluster M5 and the field stars used for the application of the procedure with 2MASS.

\begin{tabular}{|c|c|c|c|c|c|c|c|c|}
\hline \multicolumn{5}{|c|}{ M5 } & & & & \\
\hline $\begin{array}{l}V \\
(\mathrm{mag})\end{array}$ & $\begin{array}{l}V-J \\
(\mathrm{mag})\end{array}$ & $\begin{array}{c}J \\
(\mathrm{mag})\end{array}$ & $\begin{array}{c}(V-J)_{0} \\
(\mathrm{mag})\end{array}$ & $\begin{array}{c}J_{0} \\
(\mathrm{mag})\end{array}$ & & & & \\
\hline 21.95 & 2.01 & 19.94 & 1.940 & 19.914 & & & & \\
\hline 21.84 & 1.96 & 19.88 & 1.890 & 19.854 & & & & \\
\hline 21.60 & 1.84 & 19.76 & 1.770 & 19.734 & & & & \\
\hline 21.37 & 1.74 & 19.63 & 1.670 & 19.604 & & & & \\
\hline 21.16 & 1.65 & 19.51 & 1.580 & 19.484 & & & & \\
\hline 20.95 & 1.56 & 19.39 & 1.490 & 19.364 & & & & \\
\hline 20.75 & 1.50 & 19.25 & 1.430 & 19.224 & & & & \\
\hline 20.55 & 1.43 & 19.12 & 1.360 & 19.094 & & & & \\
\hline 20.36 & 1.36 & 19.00 & 1.290 & 18.974 & & & & \\
\hline 20.16 & 1.30 & 18.86 & 1.230 & 18.834 & & & & \\
\hline 19.96 & 1.26 & 18.70 & 1.190 & 18.674 & & & & \\
\hline 19.75 & 1.21 & 18.54 & 1.140 & 18.514 & & & & \\
\hline 19.53 & 1.17 & 18.36 & 1.100 & 18.334 & & & & \\
\hline 19.31 & 1.12 & 18.19 & 1.050 & 18.164 & & & & \\
\hline 19.07 & 1.08 & 17.99 & 1.010 & 17.964 & & & & \\
\hline 18.83 & 1.05 & 17.78 & 0.980 & 17.754 & & & & \\
\hline 18.59 & 1.03 & 17.56 & 0.960 & 17.534 & & & & \\
\hline \multicolumn{9}{|c|}{ Field stars } \\
\hline (1) & (2) & (3) & (4) & (5) & (6) & (7) & (8) & (9) \\
\hline HIP & $\begin{array}{c}V \\
(\mathrm{mag})\end{array}$ & $\begin{array}{c}\pi \\
(\mathrm{mas})\end{array}$ & $\begin{array}{c}\sigma_{\pi} \\
(\mathrm{mas})\end{array}$ & $\begin{array}{c}{[\mathrm{Fe} / \mathrm{H}]} \\
(\mathrm{dex})\end{array}$ & $\begin{array}{c}J \\
(\mathrm{mag})\end{array}$ & $\begin{array}{l}V-J \\
(\mathrm{mag})\end{array}$ & $\begin{array}{c}d \\
(\mathrm{pc})\end{array}$ & $\begin{array}{c}M_{J} \\
(\mathrm{mag})\end{array}$ \\
\hline 39088 & 9.239 & 19.52 & 0.83 & 0.334 & 7.806 & 1.433 & 51.2 & 4.258 \\
\hline 39342 & 7.166 & 57.88 & 0.58 & -0.043 & 5.647 & 1.519 & 17.3 & 4.460 \\
\hline 40051 & 8.778 & 29.86 & 0.82 & 0.090 & 7.131 & 1.647 & 33.5 & 4.506 \\
\hline 40419 & 8.272 & 29.39 & 1.14 & -0.483 & 6.895 & 1.377 & 34.0 & 4.236 \\
\hline 42074 & 7.330 & 45.95 & 1.01 & 0.044 & 5.917 & 1.413 & 21.8 & 4.228 \\
\hline 42281 & 8.688 & 27.02 & 1.18 & 0.310 & 7.168 & 1.520 & 37.0 & 4.326 \\
\hline 42914 & 8.183 & 32.14 & 0.82 & -0.095 & 6.832 & 1.351 & 31.1 & 4.367 \\
\hline 44341 & 8.028 & 32.18 & 1.09 & 0.210 & 6.628 & 1.400 & 31.1 & 4.166 \\
\hline 44719 & 8.410 & 25.83 & 0.91 & 0.034 & 7.014 & 1.396 & 38.7 & 4.075 \\
\hline 46580 & 7.203 & 78.87 & 1.02 & -0.110 & 5.429 & 1.774 & 12.7 & 4.914 \\
\hline 46422 & 8.855 & 25.40 & 0.83 & -0.204 & 7.348 & 1.507 & 39.4 & 4.372 \\
\hline 48754 & 8.524 & 27.18 & 1.10 & -0.321 & 7.158 & 1.366 & 36.8 & 4.329 \\
\hline 50032 & 9.068 & 23.19 & 1.09 & 0.022 & 7.538 & 1.530 & 43.1 & 4.365 \\
\hline 50274 & 8.966 & 22.42 & 0.82 & -0.246 & 7.561 & 1.405 & 44.6 & 4.314 \\
\hline 50713 & 9.360 & 17.30 & 1.26 & 0.134 & 7.971 & 1.389 & 57.8 & 4.161 \\
\hline 50782 & 7.769 & 37.30 & 1.31 & 0.063 & 6.399 & 1.370 & 26.8 & 4.258 \\
\hline 51297 & 8.859 & 29.83 & 1.03 & -0.318 & 7.379 & 1.480 & 33.5 & 4.752 \\
\hline 54538 & 9.738 & 16.39 & 1.24 & -0.156 & 8.286 & 1.452 & 61.0 & 4.359 \\
\hline 55210 & 7.275 & 45.48 & 1.00 & -0.222 & 5.914 & 1.361 & 21.9 & 4.203 \\
\hline 57321 & 9.370 & 18.94 & 1.33 & -0.073 & 7.989 & 1.381 & 52.8 & 4.376 \\
\hline 58536 & 8.411 & 27.81 & 1.05 & 0.033 & 7.034 & 1.377 & 36.0 & 4.255 \\
\hline 58949 & 8.166 & 30.58 & 1.02 & 0.008 & 6.775 & 1.391 & 32.7 & 4.202 \\
\hline 59572 & 7.918 & 32.30 & 1.02 & 0.374 & 6.571 & 1.347 & 31.0 & 4.117 \\
\hline 59639 & 8.633 & 31.54 & 0.83 & 0.027 & 7.036 & 1.597 & 31.7 & 4.530 \\
\hline 61291 & 7.143 & 61.83 & 0.63 & -0.205 & 5.626 & 1.517 & 16.2 & 4.582 \\
\hline 61998 & 8.441 & 27.45 & 1.13 & -0.242 & 7.143 & 1.298 & 36.4 & 4.336 \\
\hline 62942 & 8.247 & 38.12 & 1.44 & -0.094 & 6.661 & 1.586 & 26.2 & 4.567 \\
\hline 64103 & 9.668 & 14.41 & 1.48 & -0.290 & 8.431 & 1.237 & 69.4 & 4.224 \\
\hline 64125 & 9.402 & 19.08 & 1.28 & -0.288 & 7.882 & 1.520 & 52.4 & 4.285 \\
\hline 65121 & 8.594 & 32.83 & 1.08 & 0.142 & 6.929 & 1.665 & 30.5 & 4.510 \\
\hline 65646 & 10.773 & 18.90 & 2.38 & -0.401 & 8.865 & 1.908 & 52.9 & 5.247 \\
\hline 67344 & 8.340 & 31.78 & 1.06 & 0.052 & 6.897 & 1.443 & 31.5 & 4.408 \\
\hline 68936 & 8.356 & 26.14 & 1.18 & 0.444 & 6.952 & 1.404 & 38.3 & 4.039 \\
\hline 69075 & 9.495 & 28.79 & 1.36 & -0.490 & 7.700 & 1.795 & 34.7 & 4.996 \\
\hline 69301 & 10.760 & 15.21 & 2.44 & -0.208 & 9.219 & 1.541 & 65.7 & 5.130 \\
\hline 69357 & 7.938 & 43.35 & 1.40 & -0.081 & 6.360 & 1.578 & 23.0 & 4.545 \\
\hline 69570 & 8.235 & 27.62 & 1.12 & -0.557 & 6.992 & 1.243 & 36.2 & 4.198 \\
\hline 71673 & 10.203 & 16.33 & 1.62 & -0.182 & 8.726 & 1.477 & 61.2 & 4.791 \\
\hline
\end{tabular}


Table 17. Continued.

\begin{tabular}{|c|c|c|c|c|c|c|c|c|}
\hline \multicolumn{9}{|c|}{ Field stars } \\
\hline (1) & (2) & (3) & (4) & (5) & (6) & (7) & (8) & (9) \\
\hline HIP & $\begin{array}{c}V \\
(\mathrm{mag})\end{array}$ & $\begin{array}{c}\pi \\
\text { (mas) }\end{array}$ & $\begin{array}{c}\sigma_{\pi} \\
(\mathrm{mas})\end{array}$ & $\begin{array}{c}{[\mathrm{Fe} / \mathrm{H}]} \\
(\mathrm{dex})\end{array}$ & $\begin{array}{c}J \\
(\mathrm{mag})\end{array}$ & $\begin{array}{l}V-J \\
(\mathrm{mag})\end{array}$ & $\begin{array}{c}d \\
(\mathrm{pc})\end{array}$ & $\begin{array}{c}M_{J} \\
\text { (mag) }\end{array}$ \\
\hline 72312 & 7.761 & 50.84 & 1.04 & -0.123 & 6.151 & 1.610 & 19.7 & 4.682 \\
\hline 72339 & 8.046 & 33.60 & 1.51 & 0.133 & 6.712 & 1.334 & 29.8 & 4.344 \\
\hline 72577 & 9.073 & 32.53 & 1.56 & -0.301 & 7.313 & 1.760 & 30.7 & 4.874 \\
\hline 72688 & 7.804 & 58.96 & 1.05 & -0.027 & 5.990 & 1.814 & 17.0 & 4.843 \\
\hline 72703 & 8.380 & 25.68 & 1.29 & -0.386 & 7.118 & 1.262 & 38.9 & 4.166 \\
\hline 73547 & 7.736 & 36.84 & 0.98 & -0.522 & 6.419 & 1.317 & 27.1 & 4.251 \\
\hline 73963 & 10.343 & 13.57 & 1.91 & -0.116 & 8.770 & 1.573 & 73.7 & 4.433 \\
\hline 75266 & 8.281 & 39.35 & 1.37 & 0.155 & 6.596 & 1.685 & 25.4 & 4.571 \\
\hline 80043 & 8.901 & 38.80 & 1.37 & -0.456 & 7.116 & 1.785 & 25.8 & 5.060 \\
\hline 80700 & 8.807 & 21.50 & 1.27 & 0.314 & 7.419 & 1.388 & 46.5 & 4.081 \\
\hline 81237 & 8.756 & 25.32 & 1.15 & -0.149 & 7.369 & 1.387 & 39.5 & 4.386 \\
\hline 84164 & 9.186 & 17.74 & 1.51 & -0.304 & 7.550 & 1.636 & 56.4 & 3.795 \\
\hline 85425 & 7.879 & 32.04 & 1.24 & -0.432 & 6.591 & 1.288 & 31.2 & 4.119 \\
\hline 87089 & 8.912 & 26.16 & 1.16 & -0.112 & 7.337 & 1.575 & 38.2 & 4.425 \\
\hline 88553 & 8.460 & 26.99 & 1.19 & -0.149 & 7.146 & 1.314 & 37.1 & 4.302 \\
\hline 89497 & 8.545 & 27.11 & 1.20 & -0.088 & 7.181 & 1.364 & 36.9 & 4.347 \\
\hline
\end{tabular}

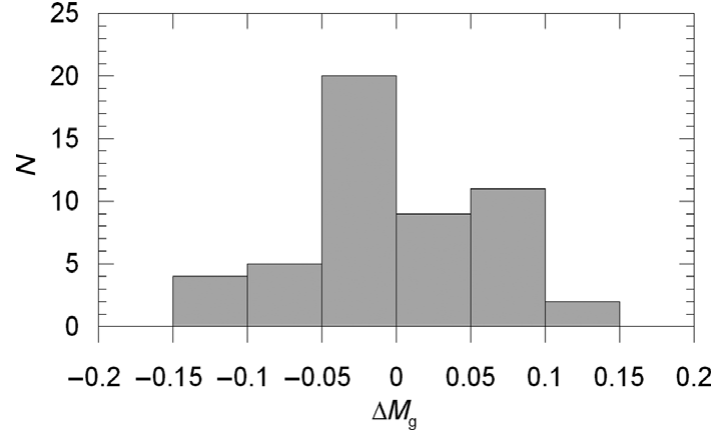

Figure 10. Histogram of the residuals.

used a set of field stars, additional to the Galactic cluster M5 $([\mathrm{Fe} / \mathrm{H}]=-1.40 \mathrm{dex})$, for the application of the $M_{J} \times[\mathrm{Fe} / \mathrm{H}]$ calibration. We tried to apply the procedure to a metal-poor cluster, i.e., M15 whose metallicity is close to the one of M92. But we noticed that the residuals were decreasing systematically from $\Delta M=0.51$ to $\Delta M=-0.26$ mag in the colour range $0.95 \leq(V-J)_{0} \leq 1.40$ mag. Comparison of the absolute magnitude-colour diagrams of the clusters M92 and M15 (Figure 11c) revealed that the reason for the systematic variation of the residuals is due to the fiducial sequence of the cluster M15. Actually, although the absolute magnitudes evaluated for M92 and M15 for the colour $(V-J)_{0}=0.95$ mag are close to each other, the absolute magnitudes of M15 deviate from the ones of M92 up to $\Delta M=0.51 \mathrm{mag}$ at redder colours. Hence, the systematic residuals in question do not originate from our procedure but they are due to the fiducial sequence of the cluster M15. The fiducial sequences of the clusters M92 and M15 are taken from the same source, i.e. Brasseur et al. (2010). The metallicities for both clusters are given as $[\mathrm{Fe} / \mathrm{H}]=-2.4 \mathrm{dex}$ in

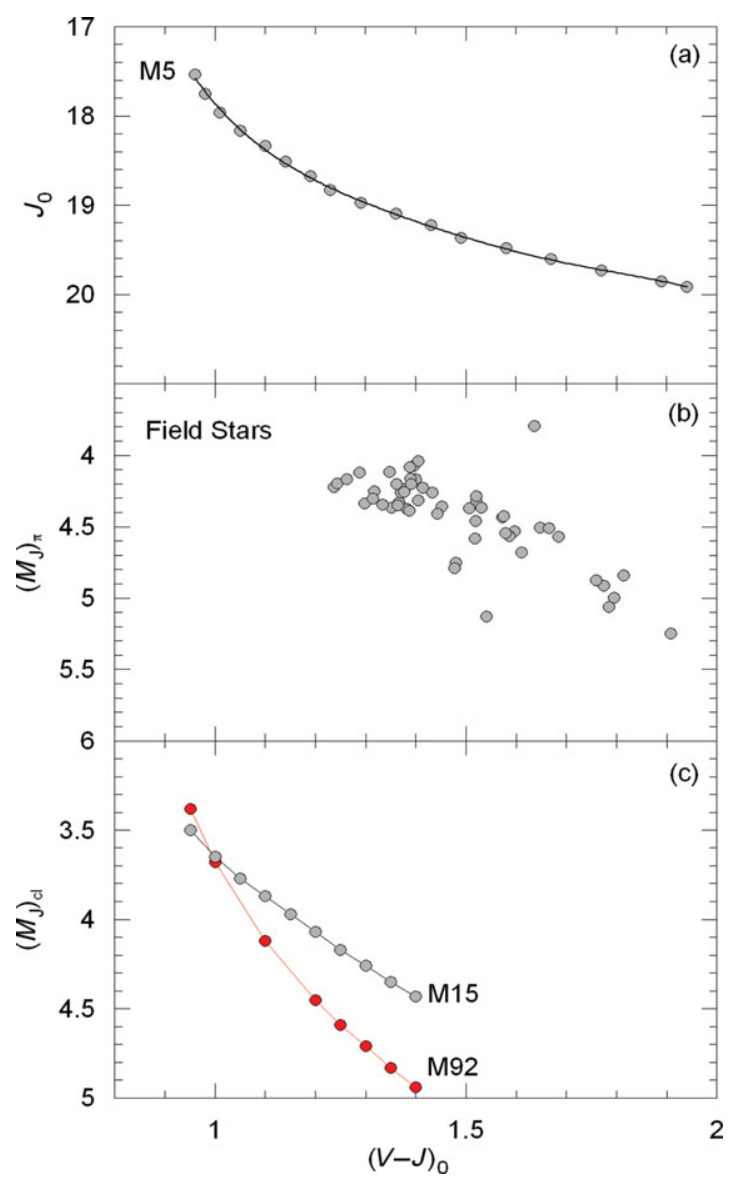

Figure 11. $J_{0} \times(V-J)_{0}$ colour-apparent magnitude diagram of the cluster M5 (panel a), distribution of the field stars with solar metallicity in the $(V-J)_{0}$ colour versus $\left(M_{J}\right)_{\pi}$ absolute magnitude diagram (panel b), and deviation of the $(V-J)_{0}$ colour $\left(M_{J}\right)_{c l}$ absolute magnitude diagram of the cluster M15 (brighter magnitudes) from the one of M92 (fainter magnitudes). 
Table 18. Absolute magnitudes $\left(M_{J}\right)_{e v}$ and residuals $\Delta M$ estimated by the procedure explained in our work. $\left(M_{J}\right)_{c l}$ and $\left(M_{J}\right)_{\pi}$ denote the absolute magnitudes evaluated by means of the colour-magnitude diagram of the M5 cluster and the parallax of the field star, respectively.

\begin{tabular}{|c|c|c|c|c|c|c|c|c|c|}
\hline \multicolumn{10}{|c|}{ Table 18a } \\
\hline $\begin{array}{l}(1) \\
(V-J)_{0} \\
(\operatorname{mag})\end{array}$ & $\begin{array}{c}(2) \\
\left(M_{J}\right)_{c l} \\
(\mathrm{mag})\end{array}$ & $\begin{array}{c}(3) \\
\left(M_{J}\right)_{e v} \\
(\mathrm{mag})\end{array}$ & $\begin{array}{c}(4) \\
\Delta M \\
(\mathrm{mag})\end{array}$ & $\begin{array}{c}(1) \\
(V-J)_{0} \\
(\mathrm{mag})\end{array}$ & $\begin{array}{c}(2) \\
\left(M_{J}\right)_{c l} \\
(\mathrm{mag})\end{array}$ & $\begin{array}{c}(3) \\
\left(M_{J}\right)_{e v} \\
(\mathrm{mag})\end{array}$ & $\begin{array}{c}(4) \\
\Delta M \\
(\mathrm{mag})\end{array}$ & & \\
\hline 0.95 & 3.11 & 3.20 & -0.09 & 1.37 & 4.74 & 4.68 & 0.06 & - & - \\
\hline 0.97 & 3.27 & 3.34 & -0.08 & 1.40 & 4.80 & 4.73 & 0.07 & - & - \\
\hline 1.00 & 3.48 & 3.52 & -0.04 & 1.43 & 4.86 & 4.79 & 0.06 & - & - \\
\hline 1.03 & 3.66 & 3.67 & -0.01 & 1.45 & 4.89 & 4.83 & 0.06 & - & - \\
\hline 1.05 & 3.77 & 3.75 & 0.02 & 1.47 & 4.93 & 4.87 & 0.06 & - & - \\
\hline 1.07 & 3.87 & 3.83 & 0.04 & 1.50 & 4.98 & 4.93 & 0.05 & - & - \\
\hline 1.10 & 4.00 & 3.93 & 0.07 & 1.53 & 5.03 & 4.98 & 0.05 & - & - \\
\hline 1.13 & 4.12 & 4.03 & 0.09 & 1.55 & 5.06 & 5.02 & 0.04 & - & - \\
\hline 1.15 & 4.19 & 4.09 & 0.10 & 1.57 & 5.09 & 5.05 & 0.04 & - & - \\
\hline 1.17 & 4.25 & 4.15 & 0.11 & 1.60 & 5.13 & 5.10 & 0.03 & - & - \\
\hline 1.20 & 4.34 & 4.27 & 0.08 & 1.63 & 5.17 & 5.15 & 0.02 & - & - \\
\hline 1.23 & 4.43 & 4.33 & 0.09 & 1.65 & 5.20 & 5.18 & 0.02 & - & - \\
\hline 1.25 & 4.48 & 4.38 & 0.10 & 1.67 & 5.22 & 5.21 & 0.01 & - & - \\
\hline 1.27 & 4.53 & 4.43 & 0.10 & 1.70 & 5.26 & 5.26 & 0.00 & - & - \\
\hline 1.30 & 4.59 & 4.50 & 0.10 & 1.73 & 5.29 & 5.30 & -0.01 & - & - \\
\hline 1.33 & 4.66 & 4.58 & 0.08 & 1.75 & 5.31 & 5.32 & -0.01 & - & - \\
\hline 1.35 & 4.70 & 4.63 & 0.07 & - & - & - & - & - & - \\
\hline \multicolumn{10}{|c|}{ Table $18 \mathrm{~b}$} \\
\hline (1) & (2) & (3) & (4) & (5) & (1) & (2) & (3) & (4) & (5) \\
\hline $\begin{array}{l}V-J \\
(\mathrm{mag}) \\
\end{array}$ & $\begin{array}{l}\left(M_{J}\right)_{\pi} \\
(\mathrm{mag}) \\
\end{array}$ & $\begin{array}{c}{[\mathrm{Fe} / \mathrm{H}]} \\
(\mathrm{dex}) \\
\end{array}$ & $\begin{array}{c}\left(M_{J}\right)_{e v} \\
(\mathrm{mag})\end{array}$ & $\begin{array}{c}\Delta M \\
(\mathrm{mag}) \\
\end{array}$ & $\begin{array}{l}V-J \\
(\mathrm{mag})\end{array}$ & $\begin{array}{l}\left(M_{J}\right)_{\pi} \\
(\mathrm{mag})\end{array}$ & $\begin{array}{c}{[\mathrm{Fe} / \mathrm{H}]} \\
(\mathrm{dex}) \\
\end{array}$ & $\begin{array}{c}\left(M_{J}\right)_{e v} \\
(\mathrm{mag})\end{array}$ & $\begin{array}{c}\Delta M \\
(\mathrm{mag}) \\
\end{array}$ \\
\hline 1.24 & 4.22 & -0.29 & 3.87 & 0.35 & 1.41 & 4.31 & -0.25 & 4.32 & -0.01 \\
\hline 1.24 & 4.20 & -0.56 & 4.01 & 0.19 & 1.41 & 4.23 & 0.04 & 4.19 & 0.04 \\
\hline 1.26 & 4.17 & -0.39 & 3.98 & 0.19 & 1.43 & 4.26 & 0.33 & 4.09 & 0.17 \\
\hline 1.29 & 4.12 & -0.43 & 4.09 & 0.03 & 1.44 & 4.41 & 0.05 & 4.25 & 0.16 \\
\hline 1.30 & 4.34 & -0.24 & 4.02 & 0.32 & 1.45 & 4.36 & -0.16 & 4.36 & 0.00 \\
\hline 1.31 & 4.30 & -0.15 & 4.01 & 0.29 & 1.48 & 4.79 & -0.18 & 4.44 & 0.35 \\
\hline 1.32 & 4.25 & -0.52 & 4.23 & 0.02 & 1.48 & 4.75 & -0.32 & 4.49 & 0.26 \\
\hline 1.33 & 4.34 & 0.13 & 3.95 & 0.39 & 1.51 & 4.37 & -0.20 & 4.51 & -0.14 \\
\hline 1.35 & 4.12 & 0.37 & 3.88 & 0.24 & 1.52 & 4.58 & -0.21 & 4.53 & 0.05 \\
\hline 1.35 & 4.37 & -0.10 & 4.12 & 0.25 & 1.52 & 4.46 & -0.04 & 4.46 & 0.00 \\
\hline 1.36 & 4.20 & -0.22 & 4.20 & 0.00 & 1.52 & 4.33 & 0.31 & 4.29 & 0.04 \\
\hline 1.36 & 4.35 & -0.09 & 4.14 & 0.21 & 1.52 & 4.28 & -0.29 & 4.57 & -0.29 \\
\hline 1.37 & 4.33 & -0.32 & 4.28 & 0.05 & 1.53 & 4.36 & 0.02 & 4.45 & -0.09 \\
\hline 1.37 & 4.26 & 0.06 & 4.09 & 0.17 & 1.54 & 5.13 & -0.21 & 4.57 & 0.56 \\
\hline 1.38 & 4.26 & 0.03 & 4.13 & 0.13 & 1.57 & 4.43 & -0.12 & 4.60 & -0.17 \\
\hline 1.38 & 4.24 & -0.48 & 4.36 & -0.12 & 1.58 & 4.43 & -0.11 & 4.61 & -0.18 \\
\hline 1.38 & 4.38 & -0.07 & 4.18 & 0.20 & 1.58 & 4.54 & -0.08 & 4.60 & -0.06 \\
\hline 1.39 & 4.39 & -0.15 & 4.23 & 0.16 & 1.59 & 4.57 & -0.09 & 4.63 & -0.06 \\
\hline 1.39 & 4.08 & 0.31 & 4.01 & 0.07 & 1.60 & 4.53 & 0.03 & 4.59 & -0.06 \\
\hline 1.39 & 4.16 & 0.13 & 4.10 & 0.06 & 1.61 & 4.68 & -0.12 & 4.68 & 0.00 \\
\hline 1.39 & 4.20 & 0.01 & 4.16 & 0.04 & 1.64 & 3.79 & -0.30 & 4.82 & -1.03 \\
\hline 1.40 & 4.07 & 0.03 & 4.17 & -0.10 & 1.65 & 4.51 & 0.09 & 4.66 & -0.15 \\
\hline 1.40 & 4.17 & 0.21 & 4.09 & 0.08 & 1.67 & 4.51 & 0.14 & 4.67 & -0.16 \\
\hline 1.40 & 4.04 & 0.44 & 3.97 & 0.07 & 1.69 & 4.57 & 0.16 & 4.71 & -0.14 \\
\hline
\end{tabular}

the same paper. Additionally, they have the same age, a bit less than 13 Gyr (Salaris, degl'Innocenti, \& Weiss 1997). Hence, the problem related to the discrepancy between the two fiducial sequences could not be solved.

We compared the absolute magnitudes estimated by the procedure explained in our paper with those evaluated either by combination of the $J_{0},(V-J)_{0}$ fiducial sequence of the cluster M5 and its true distance modulus or by the $J_{0}$ apparent magnitude and the distance of the field star in question. The total residuals lie between -0.29 and +0.39 mag. However, the range of $87 \%$ of them is rather shorter, i.e. $-0.20<\Delta M \leq+0.20 \mathrm{mag}$. The mean and the standard deviation of all residuals are $<\Delta M>=0.05$ and $\sigma=0.13$ mag. However, we state that the cluster fiducials 
Table 19. Distribution of the residuals. $N$ denotes the number of stars.

\begin{tabular}{lrr}
\hline \hline $\begin{array}{l}\Delta M_{J} \text {-interval } \\
(\mathrm{mag})\end{array}$ & $\begin{array}{c}<\Delta M_{J}> \\
(\mathrm{mag})\end{array}$ & $N$ \\
\hline$(-0.3,-0.2]$ & -0.29 & 1 \\
$(-0.2,-0.1]$ & -0.15 & 8 \\
$(-0.1,0.0]$ & -0.03 & 16 \\
$(0.0,0.1]$ & 0.06 & 36 \\
$(0.1,0.2]$ & 0.16 & 9 \\
$(0.2,0.3]$ & 0.25 & 5 \\
$(0.3,0.4]$ & 0.35 & 4 \\
\hline \hline
\end{tabular}

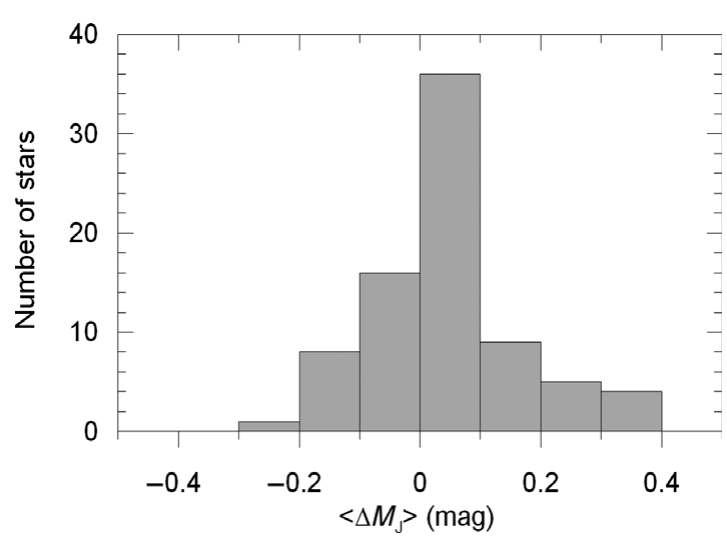

Figure 12. Histogram of the residuals.

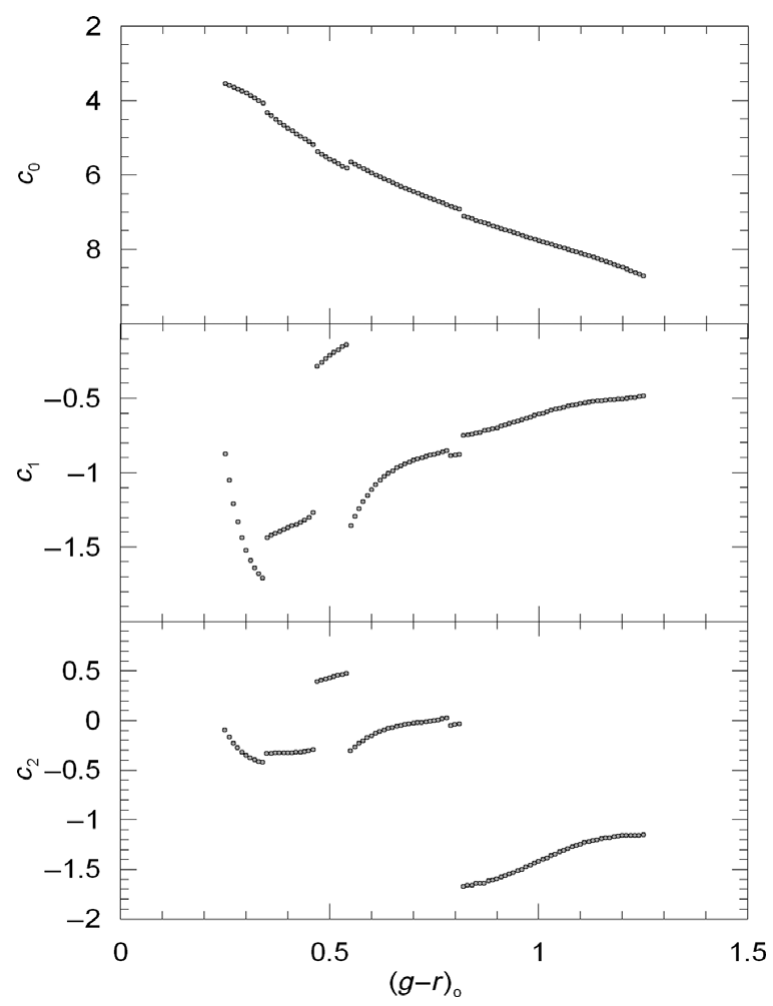

Figure 13. Variation of $c_{0}, c_{1}$, and $c_{2}$ coefficients with colour index $(g-r)_{0}$ in three panels.
Table 20. Data for two young clusters.

\begin{tabular}{lcrccc}
\hline \hline Cluster & $\begin{array}{c}E(B-V) \\
(\mathrm{mag})\end{array}$ & $\begin{array}{c}\mu_{0} \\
(\mathrm{mag})\end{array}$ & $\begin{array}{c}{[\mathrm{Fe} / \mathrm{H}]} \\
(\mathrm{dex})\end{array}$ & $\begin{array}{c}t \\
(\mathrm{Gyr})\end{array}$ & Ref \\
\hline NGC 3680 & 0.058 & 9.89 & -0.08 & 1.75 & 1,2 \\
NGC 2158 & 0.440 & 12.80 & -0.25 & 2.00 & 3,4 \\
\hline \hline
\end{tabular}

(1) Cummings et al. (2012), (2) Eggen (1969), (3) Smolinski et al. (2011), (4) Saad \& Lee (2001).

provide more accurate absolute magnitudes than the ones for the field stars. Thus, the absolute magnitude $M_{J}$ of a dwarf would be determined with an accuracy of $\Delta M<0.2$ mag. The mean and standard deviation of the residuals estimated with the same photometry $(V, J)$ for red giants were $<\Delta M>=0.137$ and $\sigma=0.080$ mag (Karaali et al. 2013b) which indicates that the absolute magnitudes of dwarfs would be determined with better accuracy. The reason of this improvement is the same as stated for the absolute magnitude $M_{g}$. The calibration of $M_{g}$ and $M_{J}$ in terms of $[\mathrm{Fe} / \mathrm{H}]$ is carried out in steps of 0.01 mag. A small step is necessary to isolate an observational error on $g-r$ and $V-J$ plus an error due to reddening. The origin of the mentioned errors shows the trend of the dwarf sequence. However, we note that the dwarf sequence is not steep. Hence, contrary to the red giant branch sequence Karaali et al. (2013a), an error in $(g-r)$ or $(V-J)$ does not imply a large change in the absolute magnitude.

The $c_{i}$ and $d_{i}(i=0,1,2)$ coefficients in Tables 9 and 12, respectively, are colour-dependent. Additionally, it seems that there is a smooth relation between a given $c_{i}$ or $d_{i}$ coefficient and the $(g-r)$ and $(V-J)$ colour. We confirmed this argument by plotting each $c_{i}$ coefficient versus $(g-r)_{0}$ colour. Actually, each panel in Figure 13 consists of a set of smooth relations with breaks at $(g-r)_{0}$ colours where the number of clusters employed in the $[\mathrm{Fe} / \mathrm{H}]$ calibrations changes. It is interesting, the mentioned breaks in the panel $c_{0}$ versus $(g-r)_{0}$ are so small that one can assume a continuous relation for the whole colour interval, i.e. $0.25 \leq(g-r)_{0}$ $\leq 1.25 \mathrm{mag}$. The mentioned relation originates from the fact that $M_{g}=c_{0}$ for $[\mathrm{Fe} / \mathrm{H}]=0 \mathrm{dex}$. 
The procedure for absolute magnitude calibration for dwarfs in Karaali et al. (2003) is based on the absolute magnitude offset which is defined as a function of ultraviolet excess. However, ultraviolet excess can not be provided easily with SDSS, especially for late-type stars, and this parameter has not been defined in 2MASS system. Whereas, the procedure in this study is metallicity-dependent, and metallicity values come from detailed analysis of individual stars using high-resolution spectroscopy in large surveys which are in operation, such as RAVE, SEGUE, GAIA-ESO, and APOGEE or which will be started in the near future, such as GALAH.

SDSS and 2MASS are two of the widely used photometric systems in the Galactic researches. Also, our procedure covers dwarfs with a large metallicity and age range. The metallicity range is $-2.15 \leq[\mathrm{Fe} / \mathrm{H}] \leq 0.37$ dex in both systems, while the age ranges are defined by the clusters used for the calibrations in SDSS and 2MASS, i.e. $4 \leq t \leq 13.2$ and $2 \leq t \leq 13.2 \mathrm{Gyr}$, respectively, where 2, 4 and 13.2 Gyr are the ages of the clusters NGC 2158, M67 and M92. The age of the cluster NGC 2158 is taken from Carraro, Girardi, \& Marigo (2002), while those for the clusters M67 and M92 were estimated in Karaali et al. (2012). Additionally, the mean residuals and standard deviations in the application of two absolute magnitude calibrations, $M_{g}$ and $M_{J}$, are small. Hence, the procedure presented in this paper can be applied with a good accuracy to large samples of dwarf stars in Galactic-structure Projects.

We stated in our previous papers (Karaali et al. 2012) that our absolute magnitude calibrations are age-restricted, i.e., they cover the stars with ages in the range defined by the youngest and oldest clusters used in the calibration. We applied the calibration $M_{g} \times[\mathrm{Fe} / \mathrm{H}]$ to two clusters, NGC 3680 and NGC 2158, younger than 4 Gyr to test the age range in the SDSS system. We used the $V$ and $B-V$ data of the cluster NGC 3680 in Kozhurina-Platais et al. (1997) and reduced them to the $g_{0}$ magnitude and $(g-r)_{0}$ colour by means of the equations of Chonis \& Gaskell (2008) given in Section 3.2.1, after necessary corrections for interstellar extinction. The colours and magnitudes of the cluster NGC 2158 taken from Smolinski et al. (2011) are already in the SDSS system. The $M_{g} \times[\mathrm{Fe} / \mathrm{H}]$ calibration could be applied to nine stars in NGC 3680 and 10 fiducials in NGC 2158, and the absolute magnitude residuals for each set of data were estimated. The mean residuals are very similar and rather high, i.e. $<\Delta M>=1.25 \mathrm{mag}$ for 9 stars and $<\Delta M>=$ $1.20 \mathrm{mag}$. for 10 fiducials, which shows that the calibration in question is limited with the age of the youngest cluster used for the calibration, i.e., 4 Gyr. The metallicities of the clusters NGC 3680 and NGC 2158 are close to the one of M67 whose age is $4 \mathrm{Gyr}$, while the ages of the first two clusters are different than the one of M67. A similar test can be carried out for the $M_{J} \times[\mathrm{Fe} / \mathrm{H}]$ calibration. Hence, we can argue that our absolute magnitude calibrations are better defined in terms of ranges in age. The colour excesses, distance moduli, metallicities, and ages of the clusters are presented in Table 20. However, the magnitudes and colours used in the evaluation of absolute magnitude residuals are not given in the paper because of space constraints.

\section{ACKNOWLEDGMENTS}

The authors would like to thank the anonymous referee who provided valuable comments and for improving the manuscript. This research has made use of NASA(National Aeronautics and Space Administration)'s Astrophysics Data System and the SIMBAD Astronomical Database, operated at CDS, Strasbourg, France.

\section{REFERENCES}

Alcaino, G., Liller, W., Alvarado, F., \& Wenderoth, E. 1990, ApJS, 72,693

An, D., et al. 2008, ApJS, 179, 326

Arp, H., \& Cuffey, J. 1962, ApJ, 136, 51

Bilir, S., Ak, S., Karaali, S., Cabrera-Lavers, A., Chonis, T. S., \& Gaskell, C. M. 2008, MNRAS, 384, 1178

Brasseur, C. M., Stetson, P. B., VandenBerg, D. A., Casagrande, L., Bono, G., \& Dall'Ora, M. 2010, AJ, 140, 1672

Breddels, M. A., et al. 2010, A\&A, 511, 90

Cardelli, J. A., Clayton, G. C., \& Mathis, J. S. 1989, ApJ, 345, 245

Carraro, G., Girardi, L., \& Marigo, P. 2002, MNRAS, 332, 705

Chen, B., et al. 2001, ApJ, 553, 184

Chonis, T. S., \& Gaskell, C. M. 2008, AJ, 135, 264

Cummings, J. D., Deliyannis, C. P., Anthony-Twarog, B., Twarog, B., \& Maderak, R. M. 2012, AJ, 144, 137

Di Criscienzo, M., Marconi, M., \& Caputo, F. 2004, ApJ, 612, 1092

Eggen, O. J. 1969, ApJ, 155, 439

ESA 1997, The Hipparcos and Tycho Catalogues, CDS/ADC Electronic Catalogues, 1239

Fan, X. 1999, AJ, 117, 2528

Ferro, A. A., et al. 2011, MNRAS, 416, 2265

Gratton, R. G., Fusi Pecci, F., Carretta, E., Clementini, G., Corsi, C. E., \& Lattanzi, M. 1997, ApJ, 491, 749

Harris, W. E. 1996, AJ, 112, 1487

Karaali, S., Bilir, S., \& Gökçe, E. Y. 2012, PASA, 29, 509

Karaali, S., Bilir, S., \& Gökçe, E. Y. 2013a, PASA, 30, 8

Karaali, S., Bilir, S., \& Gökçe, E. Y. 2013b, PASA, 30, 11

Karaali, S., Bilir, S., \& Tunçel, S. 2005, PASA, 22, 24

Karaali, S., Karataş, Y., Bilir, S., Ak, S. G., \& Hamzaoğlu, E. 2003, PASA, 20, 270

Kozhurina-Platais, V., Demarque, P., Platais, I., Orosz, J. A., \& Barnes, S. 1997, AJ, 113, 1045

Laird, J. B., Carney, B. W., \& Latham, D. W. 1988, AJ, 95, 1843

McCall, M. L. 2004, AJ, 128, 2144

Nissen, P. E., \& Schuster, W. J. 1991, A\&A, 251, 457

Phleps, S., Meisenheimer, K., Fuchs, B., \& Wolf, C. 2000, A\&A, 356, 108

Reid, I. N. 1997, AJ, 114, 161

Saad, S. M., \& Lee, S. 2001, JKAS, 34, 99

Salaris, M., degl'Innocenti, S., \& Weiss, A. 1997, ApJ, 479, 665

Sarajedini, A., Brandt, K., Grocholski, A. J., \& Tiede, G. P. 2004, AJ, 127, 991 
Sarajedini, A., Mathieu, R. D., \& Platais, I. 2003, ASSL, 289, 257

Siegel, M. H., Majewski, S. R., Reid, I. N., \& Thompson, I. B. 2002, ApJ, 578, 151

Skrutskie, M. F., et al. 2006, AJ, 131, 1163
Smolinski, J. P., et al. 2011, AJ, 141, 89

von Hippel, T., Steinhauer, A., Sarajedini, A., \& Deliyannis, C. P. 2002, AJ, 124, 1555

York, D. G., et al. 2000, AJ, 120, 1579

Zwitter, T., et al. 2010, A\&A, 522, 54 